\title{
Columnar Interactions Determine Horizontal Propagation of Recurrent Network Activity in Neocortex
}

\author{
Jason C. Wester and Diego Contreras \\ Department of Neuroscience, University of Pennsylvania Perelman School of Medicine, Philadelphia, Pennsylvania 19104
}

The cortex is organized in vertical and horizontal circuits that determine the spatiotemporal properties of distributed cortical activity. Despite detailed knowledge of synaptic interactions among individual cells in the neocortex, little is known about the rules governing interactions among local populations. Here, we used self-sustained recurrent activity generated in cortex, also known as up-states, in rat thalamocortical slices in vitro to understand interactions among laminar and horizontal circuits. By means of intracellular recordings and fast optical imaging with voltage-sensitive dyes, we show that single thalamic inputs activate the cortical column in a preferential layer 4 (L4) $\rightarrow$ layer 2/3 (L2/3) $\rightarrow$ layer 5 (L5) sequence, followed by horizontal propagation with a leading front in supragranular and infragranular layers. To understand the laminar and columnar interactions, we used focal injections of TTX to block activity in small local populations, while preserving functional connectivity in the rest of the network. We show that $\mathrm{L} 2 / 3$ alone, without underlying L5, does not generate self-sustained activity and is inefficient propagating activity horizontally. In contrast, $\mathrm{L} 5$ sustains activity in the absence of $\mathrm{L} 2 / 3$ and is necessary and sufficient to propagate activity horizontally. However, loss of L2/3 delays horizontal propagation via L5. Finally, L5 amplifies activity in L2/3. Our results show for the first time that columnar interactions between supragranular and infragranular layers are required for the normal propagation of activity in the neocortex. Our data suggest that supragranular and infragranular circuits, with their specific and complex set of inputs and outputs, work in tandem to determine the patterns of cortical activation observed in vivo.

\section{Introduction}

The neocortex is composed of local circuits heavily interconnected by vertical and horizontal projections. A generalized "canonical circuit" (for review, see Douglas and Martin, 2004) has been observed consistently across species and provides a scheme for how information may flow vertically in the cortex in response to afferent input. In primary sensory areas, thalamic input primarily to layer $4(\mathrm{~L} 4)$ is relayed to layer $2 / 3(\mathrm{~L} 2 / 3)$ and then to layer 5 (L5) and layer 6 (L6), concomitant with feedback from L5 to L2/3 and L6 to L4. Such vertical organization is linked horizontally by prominent projections within L2/3 and L5.

Embedded within this large-scale wiring diagram are local microcircuits in which neurons receive prominent input from neighboring cells (Douglas et al., 1995; Lübke et al., 2000; Feldmeyer et al., 2006; Frick et al., 2008). While the specific computational roles played by such recurrent networks are still being resolved (Pinto et al., 2003; Douglas and Martin, 2007), what is clear is that these networks provide a source of powerful local excitation and are capable of producing activity that is self-

\footnotetext{
Received Oct. 2, 2011; revised Jan. 30, 2012; accepted Feb. 28, 2012.

Author contributions: J.C.W. and D.C. designed research; J.C.W. performed research; J.C.W. analyzed data; J.C.W. and D.C. wrote the paper.

This work was supported by NIH Grant R01 EY020765 and "Training in Sleep and Sleep Disorders" Grant T32 HL07953. We acknowledge Gene Civillico for VSD analysis code and Melissa Drost for histology. We thank Larry Palmer, Cristin Welle and Ashlan Reid, Dan Denman, Leif Vigeland, and other members of the Contreras Laboratory for their helpful discussion of this work.

Correspondence should be addressed to Diego Contreras, Department of Neuroscience, University of Pennsylvania Perelman School of Medicine, 215 Stemmler Hall, Philadelphia, PA 19106-6074. E-mail: diegoc@mail.med.upenn.edu.

DOI:10.1523/JNEUROSCI.5006-11.2012

Copyright $\odot 2012$ the authors $\quad 0270-6474 / 12 / 325454-18 \$ 15.00 / 0$
}

generated and long-lasting. The strength of such recurrent circuits is highlighted under the cortical network state that occurs during slow-wave sleep, referred to as the slow oscillation. Originally described by Steriade et al. (1993a,b,c), the slow oscillation consists of alternating bouts of depolarization called "up-states" and hyperpolarization called "down-states." Importantly, the up-state represents self-sustained engagement of the entire local network in recurrent loops, including inhibitory neurons. Upstates are cortically generated, and vertical projections between layers engage circuits through the entire depth of the cortex, while horizontal projections allow the up-state to travel as a wave across the brain. Such activity potentially represents a default network state under conditions of low neuromodulatory tone, as slices of cortex will spontaneously generate up- and down-states when maintained in medium that mimics ionic concentrations measured in situ (Sanchez-Vives and McCormick, 2000).

Here, we use up-states as a tool for exploring columnar and laminar connectivity in the neocortex. We use a combination of voltage-sensitive dye (VSD) imaging, local field potentials, and intracellular recording in thalamocortical connected slices of rat barrel cortex to reveal how specific layers contribute to the initiation and propagation of self-generated recurrent activity. We found that a single thalamic input triggers an up-state that initiates within a column following a sequence of L $4 \rightarrow \mathrm{L} 2 / 3 \rightarrow \mathrm{L} 5$, which then propagates via L2/3 and L5 to neighboring columns. However, we show that L5, but not L2/3, is crucial for the spread of excitation both within a column and across columns. L5 can sustain and propagate activity to neighboring columns in the absence of L2/3. Conversely, L2/3 cannot sustain activity in the absence of the underlying L5, and often fails to allow propagation 
of activity to neighboring columns. Our data demonstrate that L5 amplifies activity in local L2/3 networks and distributes it over many columns within primary sensory cortex.

\section{Materials and Methods}

Slice preparation. Sprague Dawley rats (male) aged P14-P23 were anesthetized with $4 \%$ isoflurane and then decapitated. Brains were removed and placed in ice-cold artificial CSF (ACSF) bubbled with $95 \% \mathrm{CO}_{2} / 5 \%$ $\mathrm{O}_{2}$. Slices, $450 \mu \mathrm{m}$ thick, were cut on a Vibratome in a plane to preserve thalamocortical connections [according to Land and Kandler (2002), which is modified from the study by Agmon and Connors (1991) for juvenile rats]. Alternatively, some slices were cut in the coronal plane as a control for slice angle as discussed in Results. Slices were taken through primary somatosensory "barrel" cortex. ACSF used during the slicing procedure contained the following (in $\mathrm{mM}$ ): 252 sucrose, $3 \mathrm{KCl}, 2$ $\mathrm{MgSO}_{4}, 1.25 \mathrm{NaH}_{2} \mathrm{PO}_{4}, 26 \mathrm{NaHCO}_{3}, 2 \mathrm{CaCl}_{2}$, and 10 dextrose. Slices were transferred to a submersion holding chamber containing ACSF composed of the following (in mM): $126 \mathrm{NaCl}, 3 \mathrm{KCl}, 2 \mathrm{MgSO}_{4}, 1.25$ $\mathrm{NaH}_{2} \mathrm{PO}_{4}, 26 \mathrm{NaHCO}_{3}, 2 \mathrm{CaCl}_{2}$, and 10 dextrose. Slices were incubated in this holding chamber for $1 \mathrm{~h}$ at $32^{\circ} \mathrm{C}$ and then at room temperature until use. All experiments were approved by the University of Pennsylvania Animal Care and Use Committee.

Voltage-sensitive dye staining. Slices were stained in a static bath (with $95 \% \mathrm{CO}_{2} / 5 \% \mathrm{O}_{2}$ flowing in a closed chamber) of ACSF containing 50 $\mu \mathrm{g} / \mathrm{ml}$ di-3-ANEPPDHQ (Obaid et al., 2004) (licensed to Invitrogen) solublized in ethanol ( $0.25 \%$ ethanol by volume in the bath) for $10 \mathrm{~min}$. Di-3-ANEPPDHQ was chosen due to its low phototoxicity and low internalization (and thus high signal-to-noise ratio) (Obaid et al., 2004). After staining, slices were returned to the holding chamber to remove unbound dye before being transferred to an interface recording chamber.

Electrophysiological and optical recordings. For electrophysiological and optical recordings, slices were transferred to an interface chamber (BSC1; AutoMate Scientific) heated to $35-36^{\circ} \mathrm{C}$. Perfusion ACSF in the recording chamber was modified according to Sanchez-Vives and McCormick (2000) to promote recurrent activity and contained the following (in mM): $126 \mathrm{NaCl}, 3.5 \mathrm{KCl}, 1 \mathrm{MgSO}_{4}, 1.25 \mathrm{NaH}_{2} \mathrm{PO}_{4}, 26 \mathrm{NaHCO}_{3}, 1 \mathrm{CaCl}_{2}$, and 10 dextrose. Slices were allowed to rest for at least $1 \mathrm{~h}$ on the interface chamber in the new medium before any recordings began.

Cells were recorded intracellularly using sharp micropipettes filled with $3 \mathrm{~m}$ postassium acetate (tip resistances between 75 and $100 \mathrm{M} \Omega$ ). Pipettes were pulled using a P-97 Flaming/Brown micropipette puller (Sutter Instrument). Electrodes were advanced blindly using a Sutter MPC-200 controller with MP-285 mechanical manipulators mounted on custom-built bases angled at $50-55^{\circ}$. Recordings were made using a bridge amplifier (IR-283; Cygnus Technology) and digitized at $10 \mathrm{kHz}$ using Spike2 (CED). Current pulses were timed and controlled using a Master-8 stimulator (A.M.P.I.).

To record local field potentials (LFPs), we manufactured bipolar electrodes using tungsten electrodes with an impedance of $0.1 \mathrm{M} \Omega$ at $1 \mathrm{kHz}$ (FHC). One pole was placed in layer 1 and the other in layer 5. Signals were recorded with an A-M Systems differential AC amplifier filtered between $0.1 \mathrm{~Hz}$ and $5 \mathrm{kHz}$, and digitized at $10 \mathrm{kHz}$ using Spike2 (CED).

Optical recordings were made with a modified upright microscope (BX51; Olympus). Epiillumination was provided by a $12 \mathrm{~V}$ halogen lamp driven by a stable power supply (Kepco). Excitation light was bandpass filtered between 480 and $550 \mathrm{~nm}$ and light emitted from the slice was low-pass filtered at $590 \mathrm{~nm}$. The optical signal was collected with a CCD camera (MiCam02; BrainVision) with a sensor size of $2.9 \times 2.1 \mathrm{~mm}^{2}$ and $88 \times 60$ imageable pixels at frame rate of $1 \mathrm{~ms} /$ frame. Light was collected with a $4 \times$ objective (NA, 0.28 ; Olympus) and passed through a $0.25 \times$ demagnifier (U-TVO.25XC; Olympus) before reaching the camera, resulting in a pixel size of $\sim 33 \mu \mathrm{m}^{2}$. Optical recording was controlled with MiCam software. All data were collected as single trials with no on-line processing of $d F / F$ signals. The total recording time for each trial was $1028 \mathrm{~ms}$.

Pharmacology and drug application. Tetrodotoxin (TTX) (SigmaAldrich) was prepared as a concentrated stock in citrate buffer, $\mathrm{pH} 4.5$, and stored at $-20^{\circ} \mathrm{C}$. When needed, the stock was thawed and a solution of $20 \mu \mathrm{M}$ TTX was prepared in ACSF containing 0.15\% Coomassie blue (as a visual indicator of drug spread). This solution was loaded into a glass micropipette pulled for sharp intracellular recording with the tip broken to yield an open diameter of $\sim 10 \mu \mathrm{m}$.

TTX was pressure injected into the slice using a Picospritzer III (Parker Hannifin) with a duration of 5-10 ms at 2-4 psi. Because experiments were performed on an interface chamber, perfusion ACSF did not cover the slice, and thus the injected TTX remained within the targeted region of the cortex. If any Coomassie blue was observed in the bath after puffing (either due to the pressure being too high, or the bath level breaching the surface of the slice), the slice was discarded.

Electrical stimulation protocol. To deliver electrical stimuli, we manufactured bipolar electrodes using tungsten electrodes with an impedance of $0.1 \mathrm{M} \Omega$ at $1 \mathrm{kHz}$ (FHC). Stimuli consisted of single $100 \mu$ s pulses of 80 $\mu \mathrm{A}$ intensity in ventrobasal (VB) thalamus or $2-6 \mu \mathrm{A}$ in cortex (stimuli trains of four at $40 \mathrm{~Hz}$ were delivered to the cortex where indicated in Results). Stimulation intensity and duration were controlled with custom routines written in Igor (Wavemetrics) using NI-DAQmx Tools (National Instruments). Command voltages generated from these routines were output via a BNC-2090 (National Instruments) to a Grass stimulus isolation unit (Grass Instruments) for final current output through the bipolar electrodes.

Electrical stimuli were delivered $100 \mathrm{~ms}$ after the camera shutter opened and optical recording had begun to provide a baseline for the VSD signal before the stimulation. A set of $20-25$ such imaging trials were acquired with $10 \mathrm{~s}$ between each trial, followed by TTX injection, and then followed by acquisition of another set of 20-25 trials. Tissue health was assessed by the continued presence of spontaneous up-states in intracellular recordings or LFPs, and by the ability to evoke up-states that reached peak $d F / F$ amplitudes and durations similar to those recorded at the beginning of the experiment.

Cytochrome oxidase histology. To confirm the location of cortical layers, we revealed barrels by treating the tissue with cytochrome oxidase (CO) [according to Wong-Rilely (1979) with some modifications] In brief, after experiments, slices were fixed in $4 \%$ paraformaldehyde in 0.1 M phosphate buffer (PB) overnight. Slices were then transferred to $30 \%$ sucrose solution for $24 \mathrm{~h}$ and then resectioned at $50 \mu \mathrm{m}$ with a freezing microtome. These sections were then washed in $0.1 \mathrm{M} \mathrm{PB}$ (three times for $10 \mathrm{~min}$ each) at room temperature, incubated in a mixture of $0.1 \mathrm{M} \mathrm{PB}$ with $10 \%$ methanol and $1 \%$ hydrogen peroxide for $15 \mathrm{~min}$, and then washed again in $\mathrm{PB}$ (three times for $10 \mathrm{~min}$ each). The sections were then kept in the dark shaking overnight in $0.1 \mathrm{M} \mathrm{PB}$ containing $4 \mathrm{~g}$ of sucrose, $50 \mathrm{mg}$ of DAB (3,3'-diaminobenzidine) (Sigma-Aldrich), and $30 \mathrm{mg}$ of cytochrome oxidase (Sigma-Aldrich) per $100 \mathrm{ml}$ of PB. Finally, the sections were washed in $\mathrm{PB}$, mounted on subbed glass slides, and coverslipped. Images were acquired on an upright microscope (BX51; Olympus) using a MagnaFire camera and acquisition software (Olympus).

Image processing. Optical data consisted of differential fluorescence divided by a reference image acquired automatically by the MiCam software at the beginning of each trial $(d F / F)$. Individual trials from VSD movies were inspected separately to choose trials for averaging. Trials were screened by inspecting a region of interest (ROI) from the middle of the imaged tissue. ROIs provide an average of the $d F / F$ signal through time for pixels located under a drawn polygon (using the reference image). Only trials with a stable baseline (the period of $100 \mathrm{~ms}$ before stimulation) in which an up-state occurred were chosen for averaging. Trials in which up-states failed to be triggered generally occurred due to stimulation immediately following the end of a spontaneous up-state, which was confirmed with intracellular recordings or LFPs recorded simultaneously with imaging. For experiments with TTX, an ROI was placed on either side of the TTX to segregate trials in which an up-state was generated on one side but failed to reach the other.

Once trials were averaged, the resulting movie was postprocessed using the following steps in order. Optical data were debleached by taking a small ROI (3-5 pixels) from white matter near the internal capsule, which was smoothed by a binomial smoothing operation (a Gaussian filter, smooth factor of 50), and then subtracting this signal from the data. A $3 \times 3$ spatial filter was then applied to the data. Next, for each pixel, the 
signal was "zeroed" around the baseline through time according to the 100 ms preceding stimulation: an offset was calculated that made the average of $d F / F$ values positive to zero during this time window match the average of $d F / F$ values negative to zero. This offset was calculated for and applied to each pixel individually through time (every frame) to normalize all jitter around baseline across the entire image. Finally, a binomial smoothing operation (smooth factor of 10) was applied to the data to minimize high-frequency and high-amplitude noise peaks.

After postprocessing, the baseline noise was quantified as previously described (Civillico and Contreras, 2005, 2006). In brief, we calculated the $\mathrm{SD}$ at each pixel during the $100 \mathrm{~ms}$ before the stimulation, plotted the distribution of SDs (which was always unimodal), and chose the peak, which represents the most common SD over the image. Thresholds for signal above baseline noise were set according to this peak. For pseudocolor images, a threshold of 2-4 SDs was set depending on the width of the distribution observed qualitatively. The pseudocolor images are superimposed on a grayscale representation of the reference image. Note that this threshold is based on the most common SD across the image, so transient noise fluctuations above this threshold can occur, leading to occasional single pixel "blips" in the example images presented in Results. Finally, di-3-ANEPPDHQ reports depolarization as a decrease in fluorescence; thus, for clarity, $d F / F$ values are inverted.

Pixels located off the edge of the tissue (including pia) were deleted or blanked for clarity.

Data analysis. ROIs represent the average $d F / F$ signal of the selected area as a function of time. For analysis of the time to threshold above noise using ROIs (see Fig. 3), we chose 2 SDs as a sensitive measure of activity onset because the ROI represents further averaging of the signal and thus smoothes outliers. For analysis of propagating up-states using ROIs (see Figs. 7, 9), ROIs from each layer were taken where possible; for a minority of slices, ROIs could not be taken from all layers due to limits on the size of the imaged area.

In addition to ROIs, other analyses of the optical data were performed to characterize the activity of the slice in time and space. Line profiles produce a representation of the activity recorded at pixels under a line drawn in an arbitrary position on the reference image. Lines were 3 pixels in width, which were averaged. Pseudocolor for line profiles was set using the baseline noise measurements from the original movie as described above. Peak maps display the peak $d F / F$ amplitude recorded at each pixel from the time of stimulation until the end of the movie. For TTX experiments, subtracting the post-TTX peak map from the pre-TTX peak map provided a summary image of the region of the slice affected (see Fig. $7 B$ ). Finally, latency maps represent the time required to reach a specified threshold above baseline noise for each pixel by providing a pseudocolor for each time bin superimposed over the reference image.

To summarize monosynaptic responses to thalamic stimulation across a population of slices, we placed contour plots from multiple slices over a single reference image (see Fig. $2 D$ ). Contour plots trace the edges of contiguous pixels that reach a specified threshold. For each slice chosen for this analysis, we marked the location of each pixel reaching 3-3.5 SDs above noise with the value 1 for the frames from 0 to $10 \mathrm{~ms}$ following stimulation, and all other pixel locations with the value 0 . Contours of all contiguous pixel locations with the value 1 were then drawn over a grayscale reference. To reduce noise, contours around a single pixel that was contiguous with no others were removed. Then, the reference images with contours were overlaid such that the surface angle and curvature of each slice matched. All but one of these reference images were deleted, leaving all contour plots overlaid on a single reference. This was possible due to the fact that all images were the exact same size and pixel dimensions, and that the same region of the slice from the same region of the brain was imaged for every experiment.

To quantify the effect of TTX observed in optical recordings across experiments, we calculated how signals recorded at ROIs (for ROI locations, see Results) changed as a percentage of control conditions after TTX application within each experiment. Because TTX could delay the propagation of an up-state, we chose to compare relative peak amplitudes of the $d F / F$ signal before and after TTX. The time to peak $d F / F$ was recorded and the signal was averaged over a time window of $100 \mathrm{~ms}$ centered at this time. We took the ratio of this measurement for the post-TTX signal to the pre-TTX signal as our measure of percentage change from control. If the difference between the post-TTX and pre-TTX measurements was not $>2$ SDs above baseline noise, the ratio was forced to a value of 1 , indicating no statistically significant difference between the signals.

To quantify the effect of TTX observed in intracellular recordings across experiments, we measured the ratio of the width at half-height of the up-state after TTX to before TTX within each experiment. The up-state used for these measurements was the average of all trials for a given condition. The width at half-height was measured from visual inspection.

We determined cortical layers in VSD reference images based on observed landmarks and measurements made in cytochrome oxidasestained slices (see Fig. 2E). Layer 4 is clearly distinguished by darkly stained barrels and found at a measured depth of $\sim 500 \mu \mathrm{m}$ from the surface. Layer $2 / 3$ is a cell-dense region directly above and borders the clearly cell-sparse layer 1 . Below the layer 4 barrels is a lighter band that distinguishes layer 5A. Layer 5B and layer 6 are visible as a continuous thicker dark band below. Corresponding (but reversed) differences in coloration were observable in the grayscale reference images from optical recordings. In the reference images, layer $2 / 3$ and layer $5 \mathrm{~A}$ are darker bands that sandwich the lighter layer 4. L5B and L6 below represent a continuous lighter band. Layer 1 is also observable as a light band along the surface of the slice. Our histological observations match well those of other groups for determining cortical layers (Manns et al., 2004; Meyer et al., 2010b).

Di-3-ANEPPDHQ reports membrane depolarization as a decrease in emitted fluorescence. For clarity, in keeping with convention, we depicted depolarization as a positive change in traces for ROI analyses and warm colors in pseudocolored images in all figures.

Student's $t$ test or Wilcoxon's rank test was used for statistical tests.

All analysis was performed using custom routines written for Igor (Wavemetrics).

\section{Results}

Our goal was to use the initiation and propagation of selfsustained recurrent activity in the neocortex as a tool to investigate rules for the flow of excitation in vertical and horizontal circuits. We used thalamocortical connected slices of rat primary somatosensory barrel cortex (Agmon and Connors, 1991; Land and Kandler, 2002), maintained in medium that promotes slow oscillations, also called up- and down-states (Sanchez-Vives and McCormick, 2000). Up-states are periods of sustained depolarization due to recurrent activity generated within cortical networks. In our slices, up-states occurred either spontaneously or were triggered with a single brief $(100 \mu$ s) electrical stimulus applied to VB thalamus or different restricted layers of the cortex. The spatiotemporal properties of the responses were measured with VSD imaging, LFPs, and intracellular recordings with sharp electrodes. Contributions of different layers to the initiation and propagation of sustained activity were investigated with targeted injections of TTX. Values are mean \pm SD.

\section{Intracellular characterization of spontaneous activity and thalamic responses}

Because up-states, occurring either spontaneously or triggered by electrical stimulation, were on average longer than the time allowed by our imaging procedures, we used intracellular recordings to characterize the behavior of the slice over long time windows.

We aimed our intracellular recordings and VSD imaging to the portion of the barrel cortex overlaying the dorsal hippocampus and ventral striatum (Fig. $1 \mathrm{~A}$ ), as this region receives intact thalamic afferents from $\mathrm{VB}$ in the thalamocortical slice plane (Staiger et al., 1999). At rest, the slice generated spontaneous slow oscillations as described previously in vitro (Sanchez-Vives and 
A

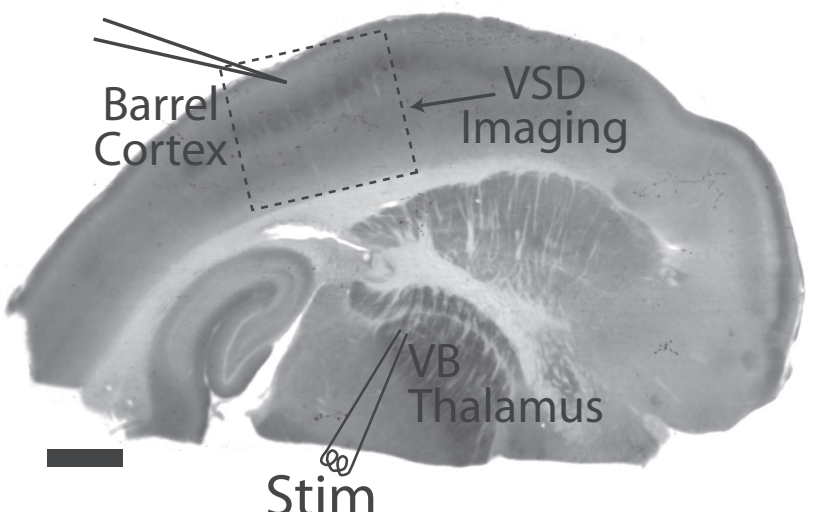

B
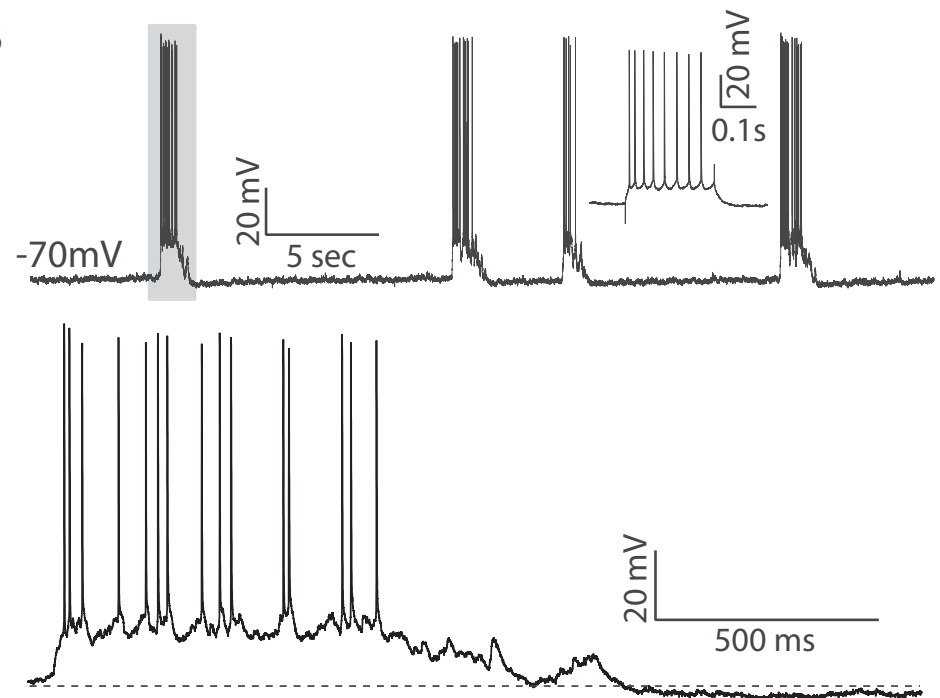

C
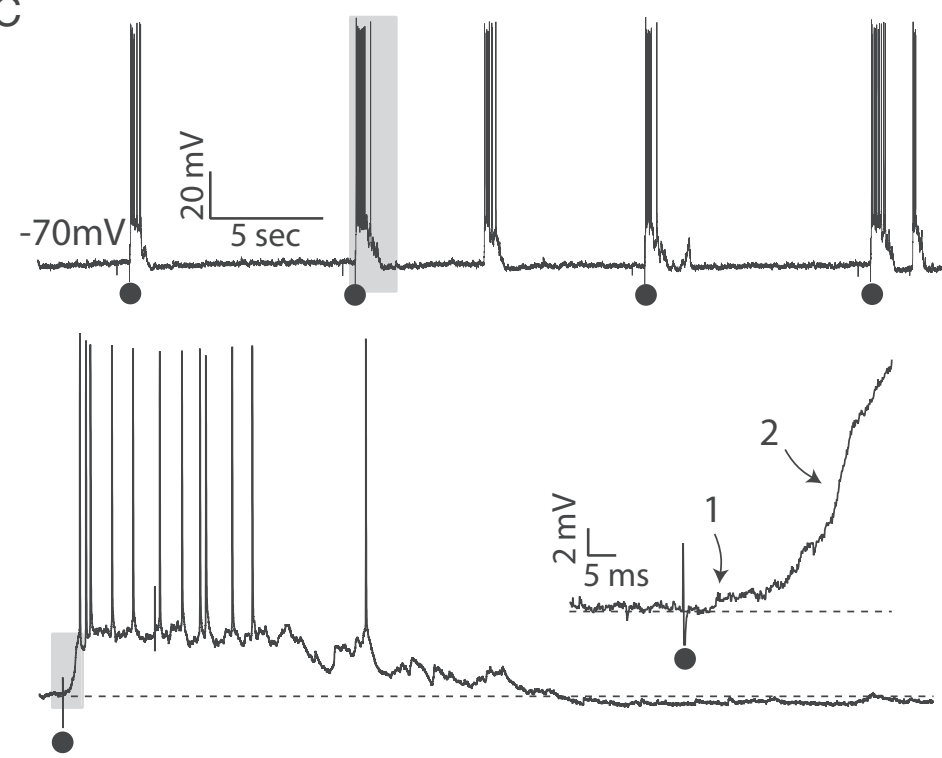

Figure 1. Intracellular recordings of slice state and responses to thalamocortical stimulation. $\boldsymbol{A}$, Cytochrome oxidase-stained thalamocortical connected slice. The dashed box indicates region of slice targeted for intracellular recordings and VSD imaging. Stimulation electrodes were placed in VB thalamus. Scale bar, $1 \mathrm{~mm}$. B, Spontaneous up-states in an example L2/3 cell (regular spiking, 200 pA injected current, inset). This slice generated spontaneous up-states with a frequency of $0.12 \mathrm{~Hz}$ (top). An expanded example (gray box) of a spontaneous up-state is shown below. C, Stimulation of VB thalamus $(80 \mu \mathrm{A}, 100 \mu \mathrm{s})$ also evoked up-states (stimulation marked by black circle). An expanded example (gray box) is shown below, with further expansion of the first $60 \mathrm{~ms}$ (inset). There are two distinct phases to the triggered up-state: an initial EPSP of $1 \mathrm{mV}$ (arrow 1) followed by the rising phase of a polysynaptic up-state (arrow 2). Because monosynaptic responses recorded in L4 always occurred with a 2-3 ms latency, we assume the initial EPSP shown here is of cortical origin, most likely L4. The duration, synaptic composition, and firing rate are similar for both evoked and spontaneous up-states.
McCormick, 2000) and similar to the original description of the slow oscillation in cortex and thalamus in vivo (Steriade et al., 1993a,b,c). All recorded cells behaved as the example L2/3 cell in Figure $1 B$ and demonstrated periods of spontaneous membrane potential $\left(V_{\mathrm{m}}\right)$ depolarization (up-states) of $10-20 \mathrm{mV}$ and lasting $992 \pm 301 \mathrm{~ms}$, occurring with a frequency of $0.05-0.12 \mathrm{~Hz}(n=10$ representative cells from layers $2 / 3,4$, and 5 from 10 slices). Brief (100 $\mu \mathrm{s})$ electrical pulses to VB (Fig. $1 B$, bottom trace, black circles) triggered up-states similar to those occurring spontaneously (duration, $989 \pm 289$ ms; same $n=10$ cells; Fig. $1 B, C$ ). Triggered up-states had a refractory period of $1 \mathrm{~s}$; thus, stimuli were delivered every $10 \mathrm{~s}$ throughout this study to ensure that full up-states were initiated. In the example cell, the synaptic response to VB stimulation had a 5 ms latency (Fig. 1C, no. 1), and was followed by the initiation of an up-state (Fig. $1 C$, no. 2). During up-states (both spontaneous and triggered), regular spiking neurons did not fire at frequencies $>25 \mathrm{~Hz}$, and fast spiking (presumably inhibitory interneurons) also participated (data not shown), reflecting that up-states do not result from pathological disinhibition, but rather a balance of excitation and inhibition (Shu et al., 2003). Across the population, cells recorded at the site of up-state initiation (i.e., responding within $20 \mathrm{~ms}$ of VB stimulation) revealed monosynaptic latencies in $\mathrm{L} 4(3.0 \pm 1.9 \mathrm{~ms} ; n=$ 43 cells, $n=37$ slices), while response latency in L2/3 was significantly longer (7.7 $\pm 4.7 \mathrm{~ms} ; n=20$ cells, $n=20$ slices) (mean \pm SD; $p<0.001$, Wilcoxon's rank test). Thus, there appeared to be an order in the activation of triggered up-states in agreement with the distribution of synaptic latencies to whisker stimulation in vivo (Wilent and Contreras, 2004).

We next used optical recordings with VSDs to map the initiation and spread of self-sustained network activity triggered by thalamic input. We hypothesized that up-states would spread in an organized manner within a barrel column and then to neighboring columns.

Combined VSD imaging and intracellular recording reveal organized initiation and propagation of thalamically triggered recurrent network activity VSD imaging provides high spatial and temporal resolution for recording membrane voltage (Cohen and Salzberg, 1978; Contreras and Llinás, 2001; Petersen and Sakmann, 2001). VSD imaging is particu- 
A
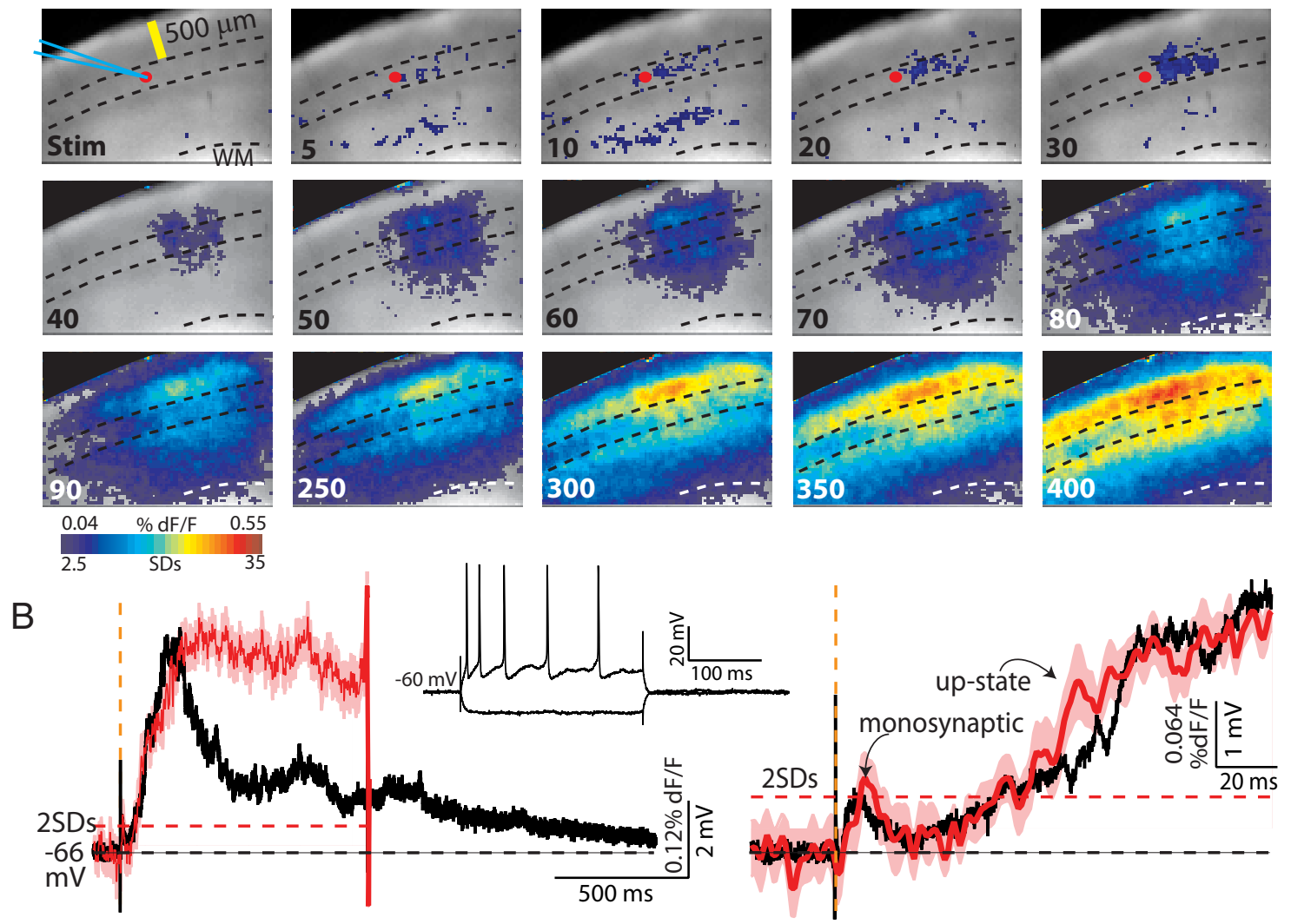

C

Latencies to $2.5 \mathrm{SDs}$ $0-100 \mathrm{~ms}$

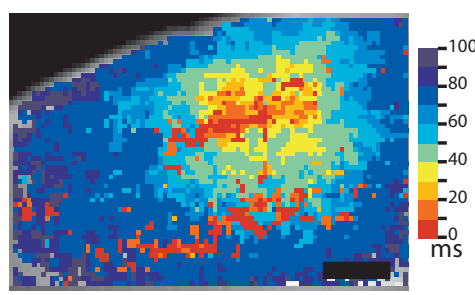

D Monosynaptic responses $\mathrm{n}=11$ slices

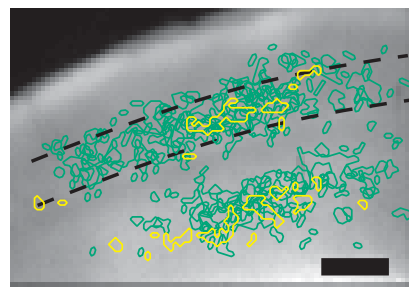

$E$

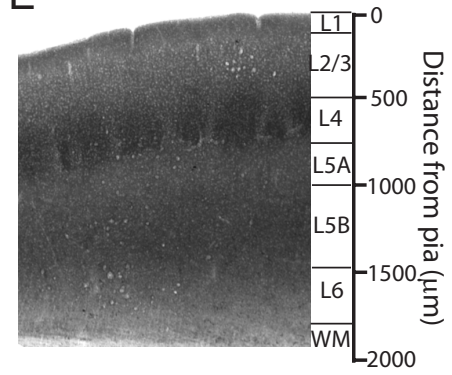

Figure 2. Responses to thalamocortical stimulation measured with VSD imaging and intracellular recording. $A$, VSD movie frames (time in milliseconds) demonstrating the response to VB stimulation. The red dot in the first row of frames represents a ROI from layer 4 in the vicinity of an intracellular recording. The dashed lines denote the borders of $L 4$ as measured from the surface of the slice (see $\boldsymbol{E}$ below), and white matter is denoted by "WM." The movie is an average of 17 single trials. Color scale provides values in both percentage $d F / F$ and SDs above baseline noise (see Materials and Methods). $\boldsymbol{B}$, Averaged intracellular recording from L4 (regular spiking cell, $325 \mathrm{pA}$ current pulse, inset) and VSD signals from the ROI ( $\boldsymbol{A}$, red dot). Close-up of the initial part of the response is shown at right. The $L 4$ cell was recorded immediately adjacent to, but not at, the site of up-state initiation. A small monosynaptic response was recorded in the $L 4$ cell and at the $L 4$ (red) $\mathrm{ROI}$ (ROI trace is mean \pm SEM). The monosynaptic thalamocortical response recorded in the cell and with VSD returns to baseline before the up-state that was initiated in the neighboring column reaches this part of the slice. Note match of VSD and intracellular measurements. The horizontal red dashed line indicates threshold to 2 SDs above baseline noise (see Materials and Methods). The vertical orange dashed line indicates time of thalamic stimulation. C, Latency map of response to thalamic input. Color scale represents time to 2.5 SDs above baseline noise from the time of VB stimulation to $100 \mathrm{~ms}$, at $10 \mathrm{~ms}$ per bin. Scale bar, $500 \mu \mathrm{m}$. D, Contour plots of monosynaptic responses to VB stimulation from 11 slices overlaid on a single reference image (see Materials and Methods). Monosynaptic responses are clustered in L4 and L5B/6. The yellow contours are from the slice in part $\boldsymbol{A}$ above. Scale bar, $500 \mu \mathrm{m}$. $\boldsymbol{E}$, Histology for the slice from $\boldsymbol{A}-\boldsymbol{D}$ stained for cytochrome oxidase to reveal barrels and layering.

larly powerful in the slice preparation in which all cortical layers are visible, as well as multiple cortical columns. We recorded cortical responses to a single thalamic stimulus with a resolution of $33 \mu \mathrm{m}^{2} /$ pixel and $1 \mathrm{~ms} /$ frame in slices spontaneously generating up- and down-states (Fig. 2; for imaged region, see Fig. 1A). In all VSD movie examples, pseudocolor represents significant depolarization relative to the baseline (see Materials and Methods), and responses in blue represent at least $1 \mathrm{mV}$ of depolarization (Fig. $2 \mathrm{~B}$ ). In the representative example of Figure 2, CO staining allowed for the measurement of cortical depth and the delineation of layers (Fig. 2E). Barrels were clearly visible $500 \mu \mathrm{m}$ below the pial surface. We called L5A the lighter band immediately below the barrels, L5B the darker band below L5A, and L6 the lighter band above the white matter (Manns et al., 2004; Meyer et al., 2010b).

In the example movie frames in Figure $2 A$, at 5 ms a monosynaptic response was visible in $\mathrm{L} 4$ and $\mathrm{L} 5 \mathrm{~B} / 6$ [as observed in vivo by de Kock et al. (2007) and in agreement with Agmon and Connors (1992), Laaris et al. (2000), Wimmer et al. (2010), and Meyer et al. (2010a,b)]. The monosynaptic response in L4 accu- 
rately represented underlying neuronal depolarization, as shown by comparing the $d F / F$ signal from a small ROI (see Materials and Methods) around a simultaneously recorded neuron in L4 (Fig. $2 B)$. Activity in $\mathrm{L} 4$ adjacent to the recorded neuron representing two barrels ( $~ 500 \mu \mathrm{m}$ of tissue) persisted, giving rise to an upstate that invaded L2/3 within 20-30 ms, L5A $10 \mathrm{~ms}$ later, and then spreading vertically through the entire column. The up-state propagated horizontally through L $2 / 3$ and L5, reaching the intracellular recording site and ROI by $50 \mathrm{~ms}$ (Fig. $2 \mathrm{~B}$, right). The up-state filled the entire imaged area by $250 \mathrm{~ms}$, indicating depolarization of all layers (L1 through L6). For the remainder of the imaging time (up to $1000 \mathrm{~ms}$ ), the spatial pattern of activation did not change, but the simultaneously recorded neuron repolarized to baseline after $1000 \mathrm{~ms}$ (Fig. $2 \mathrm{~B}$, black trace). The sequence of activation illustrated by the still frames is clearly visible in the latency map (Fig. 2C), which shows each pixel color coded by the time to crossing 2.5 SD above baseline noise (see Materials and Methods). Although small but physiologically relevant depolarization is potentially lost below noise threshold, our simultaneous intracellular recording and VSD imaging (Figs. $2 B, 3 B$ ) demonstrate that the dynamics of small subthreshold depolarizations were faithfully captured.

The location of monosynaptic responses was consistent across experiments, as shown by plotting their contours (values of $d F / F$ $>3$ SDs of baseline noise, integrated between 1 and 5-10 ms) for 11 slices aligned and superimposed on a single reference image (Fig. 2D) (see Materials and Methods). This is also in agreement with the laminar profile of monosynaptic responses in mouse thalamocortical slices observed with VSD imaging by Laaris et al. (2000). Although monosynaptic responses in L4 can be seen in multiple barrels, activity in only one or two barrel columns initiated an up-state. We term such a column a "primary column," and one to which an up-state propagates an "adjacent column." The term "column" is used here only to emphasize the vertical character of the initial activation and of the advancing front, without implying knowledge of borders of individual columns either anatomically or functionally.

In a small number of slices $(n=6)$, the response to a single thalamic stimulus was widespread and led to the generation of up-states from multiple sites (data not shown). These slices likely contain significant input from the posterior medial thalamic nucleus (POm) in addition to $\mathrm{VB}$, leading to simultaneous activation of septal and barrel circuits in various layers. We chose to focus on responses resulting from stimulation primarily of VB, which resulted in clearly localized sites of up-state initiation. Thus, these data are no longer considered in the present study.

To visualize the pattern of vertical propagation and reveal the difference between primary and adjacent columns, we constructed line profiles (Fig. 3A1), which represent the average $d F / F$ signal as a function of time from pixels falling along an arbitrary line drawn over the slice. The primary column line profile (Fig. $3 A 1$, top panel) confirmed the sequence of activation observed in the single frames [i.e., L4 $\rightarrow \mathrm{L} 2 / 3$ (down arrow at $30 \mathrm{~ms}$ ) $\rightarrow$ L5A (up arrow at $40 \mathrm{~ms}$ )]. Because VSD stains not just somata but also axons and dendrites, a signal recorded in L4 has the potential to include L5 apical dendrites as a source. This is undoubtedly true after an up-state has been initiated. However, the monosynaptic signal in L4 before up-state initiation persists for tens of milliseconds before detection in L5A; thus, we exclude L5 as a source of this initial response. The adjacent column (Fig. 3A1, bottom panel) showed near simultaneous vertical activation with a latency of $75 \mathrm{~ms}$, indicating a propagation velocity of $20 \mathrm{~mm} / \mathrm{s}$. This velocity is slightly faster than reported previously $[10 \mathrm{~mm} / \mathrm{s}$ by Sanchez-Vives and McCormick (2000)]; however, that measurement was in relation to spiking, whereas our measurement is to subthreshold depolarization. Also, this velocity is still slower than reported in vivo (up to $100 \mathrm{~mm} / \mathrm{s}$ ) (Amzica and Steriade, 1995).

To quantify the vertical flow of depolarization for the population, we used ROI analysis (Fig. 3A2, inset). ROIs (L2/3, L4, $\mathrm{L} 5 \mathrm{~A} / \mathrm{B}, \mathrm{L} 5 \mathrm{~B} / 6$ ) were placed according to depth from the surface of the slice and by the layering apparent in the reference image, in the $d F / F$ signals during up-states, and under light microscopy. The borders of L5B and 6 were not reliably distinguishable and are thus represented by the joint ROI "L5B/6." Similarly, we chose to label the upper L5 ROI "L5A/B" because we could not discount small contributions from layer $5 \mathrm{~B}$. The ROIs from the experiment above clearly distinguished the sequential activation of layers in the primary column (Fig. 3A2, top) and the nearsimultaneous activation in the adjacent column (Fig. 3A2, bottom). ROIs from two additional experiments (Fig. $3 B$ ) in which we also obtained intracellular recordings in L2/3 (Fig. $3 B$, top row) and L5A (Fig. 3B, bottom row) confirmed the order of activation in the primary column. The precise matching of the intracellular responses with the corresponding fluorescence signal from the same layer validates the precision and reliability of our ROI measurements. Indeed, the mean latency of the $d F / F$ signal to VB responses in L4 (measured 2 SDs above noise; $n=18$ slices) was $6.9 \pm 5.9 \mathrm{~ms}$, while the latency to reach $2 \mathrm{mV}$ of depolarization (comparable with the 2 SD fluorescence threshold) in the intracellular recordings from L4 was $5.3 \pm 3.2 \mathrm{~ms}(n=$ 43 cells from 37 slices). The same close matching was true in $\mathrm{L} 2 / 3$ $\left(d F / F=22.22 \pm 11.95 \mathrm{~ms} ; V_{\mathrm{m}}=16.3 \pm 11.1 \mathrm{~ms} ; n=25\right.$ cells from 25 slices). To compare between layers, we normalized ROI latencies to $\mathrm{L} 2 / 3$ and found that the monosynaptic activation of $\mathrm{L} 4$ preceded activation of $\mathrm{L} 2 / 3$ by $16.6 \pm 8.1 \mathrm{~ms}$ (mean $\pm \mathrm{SD} ; p<$ 0.01 ), which in turn preceded L5A/B by $8.1 \pm 8.2 \mathrm{~ms}$ (Fig. $3 C$; $p<$ 0.01 ; two lowest data points not included in mean since they represent monosynaptic activation). The L5B/6 ROI included slices with monosynaptic responses and those without, thus generating too much variability to be interpreted (but see below for 5 SDs). In the adjacent column, latencies to 2 SDs above threshold revealed no specific sequence of activation (data not shown).

To quantify the propagation of the up-state, we raised the latency threshold to 5 SDs to include only strong depolarization (Fig. 3C). We found that $\mathrm{L} 4$ and $\mathrm{L} 2 / 3$ in the primary column reached threshold at the same time and earlier than $\mathrm{L} 5 \mathrm{~A} / \mathrm{B}$ $(12.9 \pm 13.2 \mathrm{~ms} ; p<0.01)$ and L5B/6 (30.2 $\pm 28.1 \mathrm{~ms} ; p<0.01)$. In contrast, in the adjacent column, the up-state invaded $\mathrm{L} 2 / 3$ and L5A/B at the same time, and before both L4 (11.8 $\pm 9.1 \mathrm{~ms}$; $p<0.01)$ and L5B/6 (14.6 $\pm 18.1 \mathrm{~ms} ; p<0.01)$. This indicates that $\mathrm{L} 2 / 3$ and $\mathrm{L} 5 \mathrm{~A} / \mathrm{B}$ are critical for the propagation of up-states in adjacent columns, but does not reveal their relative importance.

To determine the role of supragranular and infragranular layers in initiation and propagation of up-states, we used directed injections of TTX concomitant with imaging, LFPs, and intracellular recordings.

\section{Injection of TTX into L2/3 can stop up-state initiation from VB input}

Injections of TTX $(<1 \mu \mathrm{l}, 20 \mu \mathrm{M}$ plus $0.15 \%$ Coomassie blue to visualize the injection site) into L2/3 (see Materials and Methods) were approximately circular with a diameter of $400-500 \mu \mathrm{m}$, which represents one to two columns. LFP recordings confirmed the lack of activity at the site of the injection (data not shown) and 

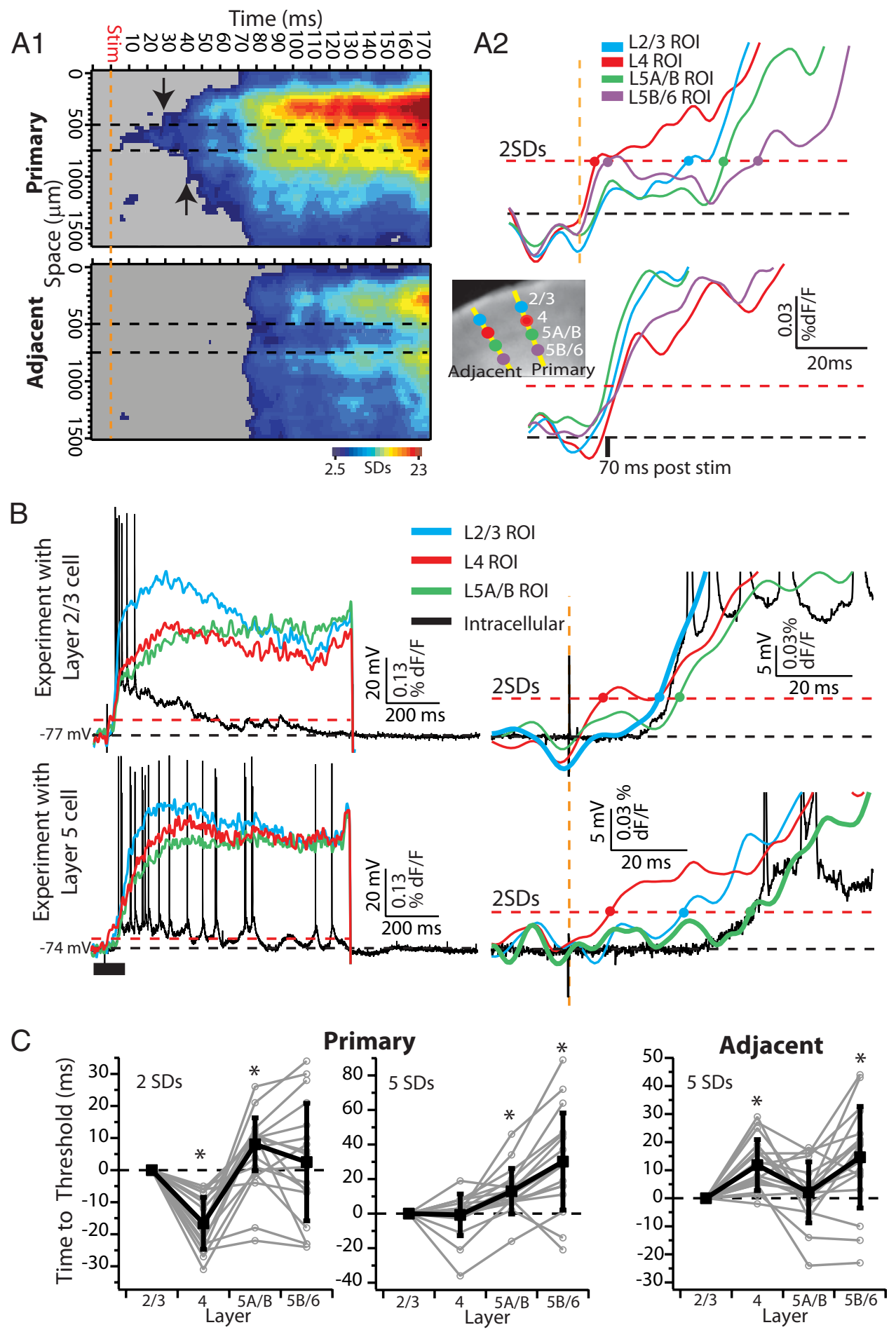

Figure 3. Analysis and quantification of thalamocortical responses recorded with VSD imaging. A, Line profiles and ROl analyses for the slice in Figure 2.A1, Line profiles through the primary (top) and adjacent (bottom) columns. The $0 \mu \mathrm{m}$ represents the start of the line at the surface of the slice, and time represents the time poststimulation. The borders of $L 4$ are bracketed by the dashed black lines and are based on measured depth from the surface. The downward arrow in the primary column line profile indicates activity invading L2/3, and the upward arrow indicates activity invading L5. The up-state travels as a wave with a single front to the adjacent column. $\mathbf{A} 2$, ROls through the primary (top) and adjacent (bottom) columns. The black dashed line indicates the baseline, and the red dashed line indicates 2 SDs above baseline noise. The time to 2 SDs above baseline noise is indicated with colored dots for each ROI. The placement of line profiles and ROls is shown over a grayscale reference image. Time of VB stimulation is marked by the dashed orange lines in all. $\boldsymbol{B}$, Examples from two additional slices of up-state initiation in a primary column. ROls were taken perpendicular to the surface of the slice as in part $A$, here centered on the site of the intracellular recording. A L2/3 cell was recorded in the top example, and a $L 5$ cell was recorded in the bottom example. An expansion of the initial part of the response is provided at right. The time to 2 SDs above baseline noise is indicated with dots for each ROl. Note in both cases that a $L 4 \rightarrow L 2 / 3 \rightarrow L 5$ order of activation of the primary column is apparent. Also note agreement of intracellular and VSD recordings. ROls are from averages of multiple trials, while the intracellular recording is a representative single trial. C, Quantification of the order of activation of signals from ROls representing cortical layers for a population of slices $(n=18)$. The $y$-axis represents the time from VB stimulation to dF/Fsignal crossing a threshold above baseline noise (2SDs for initial response or 5 SDs for an up-state in progress), normalized to the time measured in L2/3. Individual trials are shown in gray, and the mean $\pm S D$, in black. ${ }^{*} p<0.05$, Student's $t$ test for difference from 0 . Values are provided in text. 
A
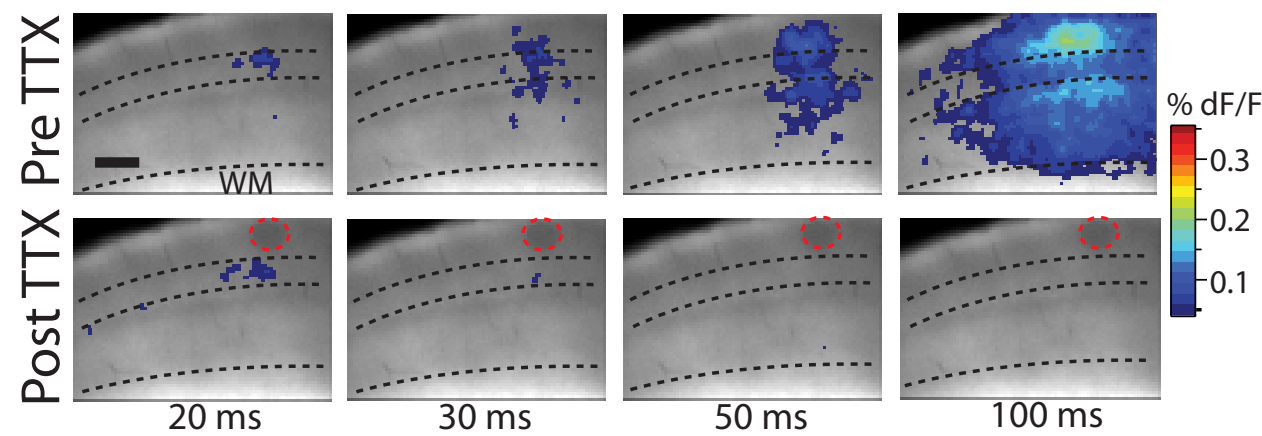

B

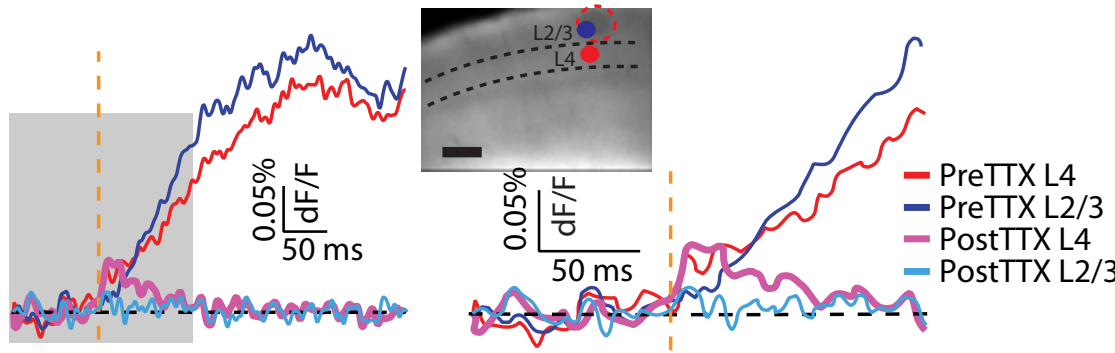

C
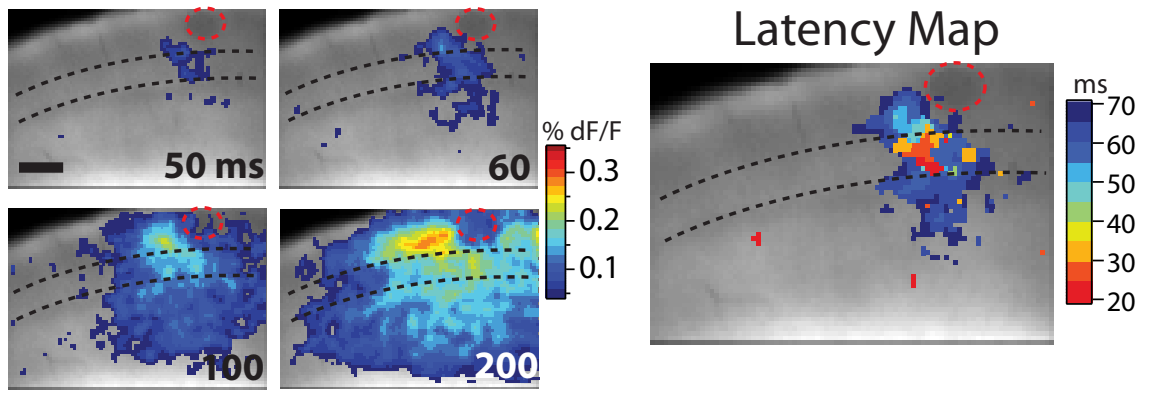

Figure 4. TTX injection into L2/3 can block up-states triggered by thalamic input. $A$, VSD movie frames before and after TTX injection (red dashed circle) in L2/3. The bottom row is the average of the 18 of 25 trials in which VB stimulation failed to trigger an up-state. Scale bar, $500 \mu \mathrm{m} . \boldsymbol{B}, \mathrm{ROls}$ at the site of the TTX injection (L2/3) and in L4 immediately below for the trials in part $A$. After TTX injection, the monosynaptic response in L4 is preserved. $C$, In 7 of 25 trials, an up-state was triggered. VSD movie frames are shown at left. On the right is a latency map showing spread of excitation into L2/3 to the immediate left of the TTX injection site, followed by columnar activation.

both LFP and intracellular recordings confirmed the lack of effect of TTX outside the borders discernible with the dye (see Figs. 5, 6, 11).

An example is shown in Figure 4 in which TTX was targeted to $\mathrm{L} 2 / 3$ precisely above the monosynaptic response to VB stimulation. The injection blocked up-state initiation in the majority of trials ( 18 of 25; Fig. $4 A$ ), even though the monosynaptic L4 response was preserved as shown by comparing the amplitude of ROIs relative to control (Fig. $4 B$ ). In a minority of trials ( 7 of 25), activity invaded L2/3 immediately adjacent to the TTX injection site, followed shortly by invasion of L5 (see latency map in Fig. $4 C$ ) and by the generation of an up-state indistinguishable from control (Fig. 4C). This result suggests that transmission of excitation from L4 to L2/3 is important for up-state initiation but does not rule out the importance of L5 excitation. Furthermore, because responses to thalamic stimulation commonly included other barrels and deeper layers, the effect of the L2/3 TTX injections was difficult to interpret, as up-states still occurred in some or all trials ( $n=4$ of 5 slices). Therefore, we repeated the experiments with TTX injections but used localized direct cortical stimulation.

\section{L5 but not L2/3 is necessary for up-state initiation}

To determine the relative roles of supragranular and infragranular layers in up-state initiation, we used minimal electrical stimulation that was capable of triggering an up-state in every trial, applied directly to the cortical layer. We monitored the occurrence of up-states with an LFP recorded several hundred micrometers from the stimulation site (observable in the Fig. 5 reference images). L2/3 minimal stimulation $(5.5 \pm 1.6 \mu \mathrm{A}$; $100 \mu \mathrm{s} ; n=10$ slices; Fig. 5) resulted in local activation followed shortly by the deeper layers, and then a propagating up-state (Fig. 5A). The delay to activate infragranular layers was quantified by ROI analysis; the time to 2 SDs in L5A/B followed L $2 / 3$ by $7 \pm 5 \mathrm{~ms}(p<0.05 ; n=10$ slices; Fig. $5 B)$, suggesting deeper layers were not being directly stimulated. When TTX was injected into upper L5 (Fig. 5C), stimuli in L2/3 failed to trigger an up-state in all trials even when the stimulus consisted of trains of four stimuli at $40 \mathrm{~Hz}$, which activated a much larger area in L2/3 and often included L4 ( $n=4 / 4$ slices) (Fig. $5 C$ ). TTX injection did not block spontaneous up-states recorded in the LFP ( $n=4$ of 4 slices) (Fig. 5D). 
A
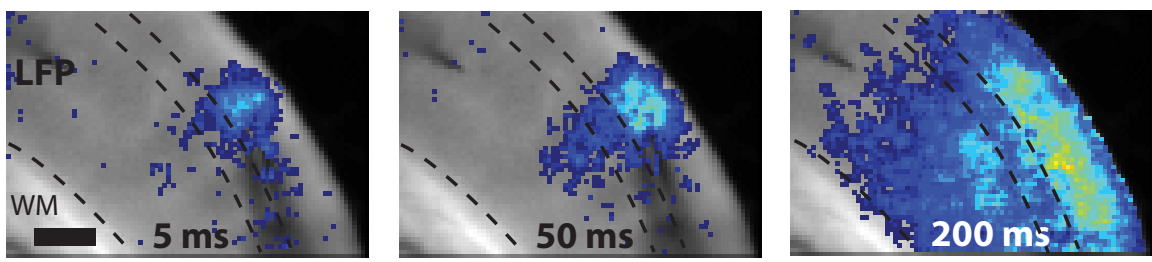

B
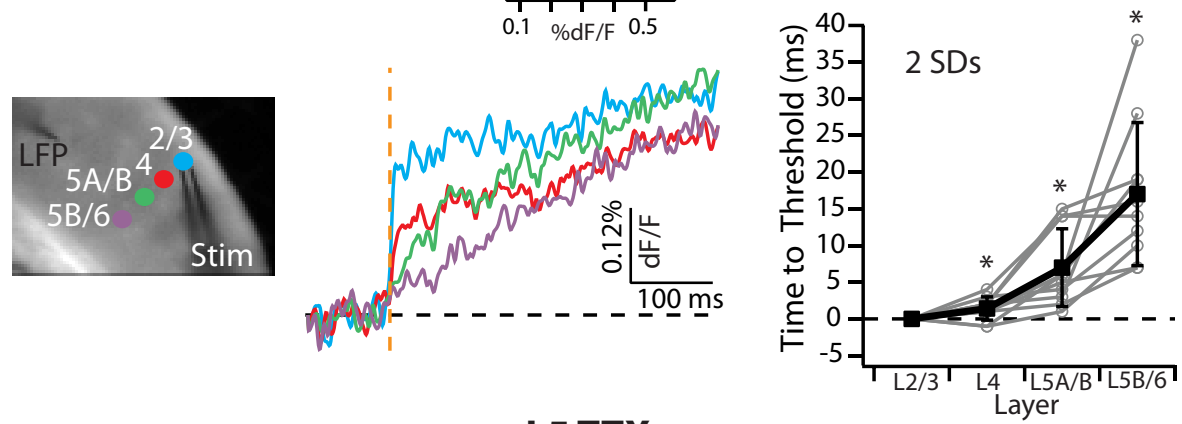

C

\section{L5 TTX}
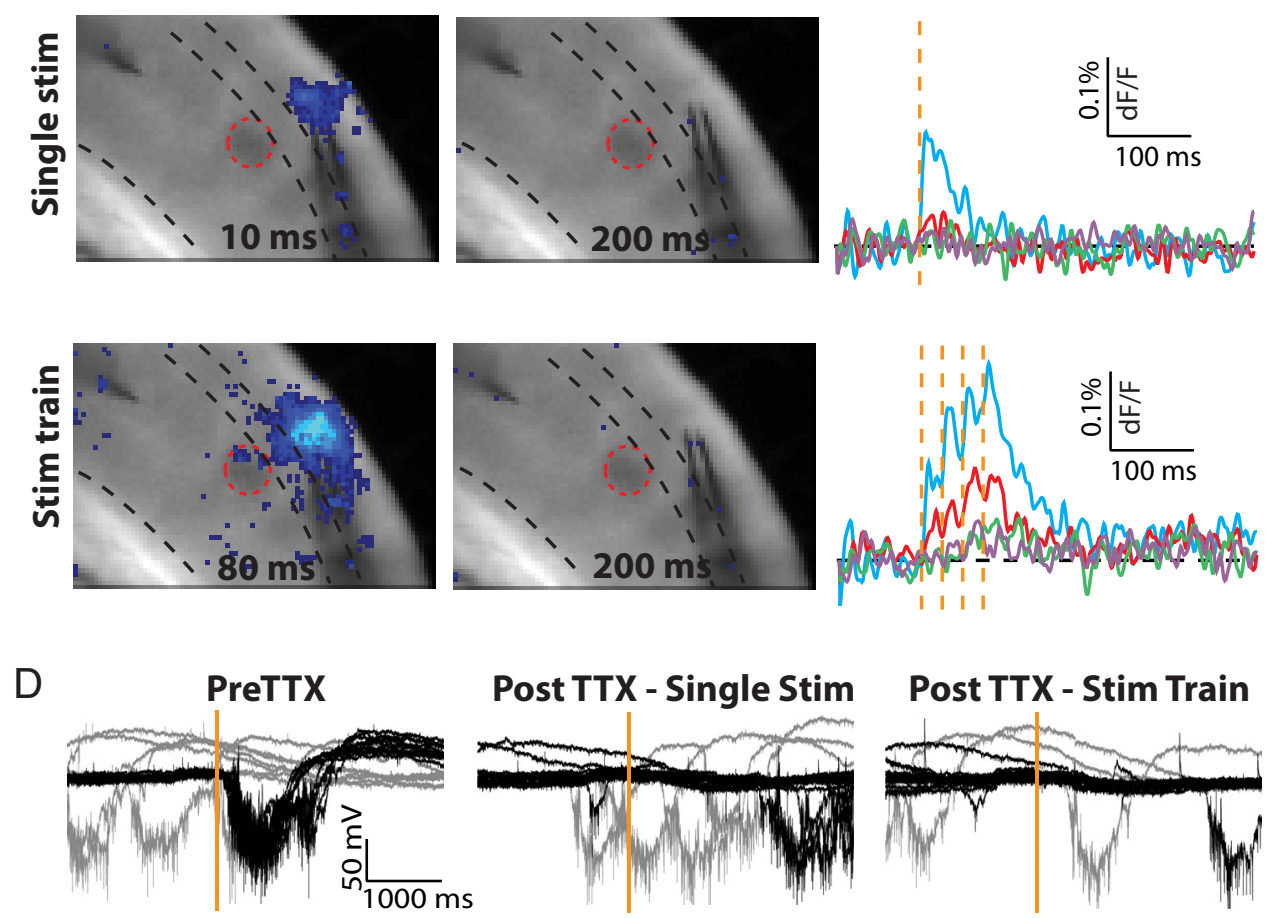

Figure 5. TTXinjection in L5 blocks up-state initiation triggered by L2/3 stimulation. $A, V S D$ movie frames demonstrating response to L2/3 stimulation ( $5 \mu A, 100 \mu \mathrm{s})$. The response starts in L2/3, then activates a column, and then spreads across the cortex. Times are poststimulus. Scale bar, $500 \mu \mathrm{m}$. B, Left, ROIs through the depth of the cortex aligned vertically to the stimulation site. Right, The time to reach 2 SDs above baseline noise was measured and then normalized to the time reported for the L2/3 ROI. Across the population, activation proceeded from L2/3 to deeper layers. Individual trials are shown in gray, and the mean $\pm S D$, in black. ${ }^{*} p<0.05$, Student's $t$ test for difference from $0 . C$, TTXinjection into $L 5$ (red dashed circle) aligned to the stimulating electrode blocks up-state initiation in response to a single stimulus (top row) and a train of four stimuli at $40 \mathrm{~Hz}$ (bottom row). Times are poststimulus. The traces on the right are from the same ROIs as in part $\boldsymbol{B}$ above. The dashed vertical orange lines denote time of individual stimuli. $\boldsymbol{D}$, LFP recording (see $\boldsymbol{A}$ and $\boldsymbol{B}$ above for labeled position in reference image). Shown are individual trials aligned to the stimulation time (orange line). The black traces represent trials in which stimuli were delivered during a down-state, and are the same trials averaged in the VSD movies above. Trials in which a spontaneous up-state coincided with the stimulation are shown in gray and are thus not included in the average VSD movie. Note the occurrence of spontaneous up-states recorded after TTX injection.

In the inverse experiment, L5 minimal stimulation $(5 \pm 1.2$ $\mu \mathrm{A} ; 100 \mu \mathrm{s} ; n=7$ slices) resulted in local activation followed by $\mathrm{L} 2 / 3$ (time to $2 \mathrm{SDs}$ in $\mathrm{L} 5 \mathrm{~A} / \mathrm{B}$ preceded L2/3 by $2.6 \pm 2.5 \mathrm{~ms} ; p<$ $0.05 ; n=7$ slices) and by the generation of a control up-state (Fig. $6 \mathrm{~A}$, top row). However, in stark contrast with $\mathrm{L} 2 / 3$ stimulation, TTX injected into L2/3 above the stimulation site did not block up-state initiation by single stimuli in any trials ( $n=4$ of 4 slices;
Fig. $6 A$, bottom row). In the example in Figure 6, two TTX injections were made in $\mathrm{L} 2 / 3$, affecting $\sim 850 \mu \mathrm{m}$ (Fig. $6 B$ ). A single stimulus resulted in activity that was sustained in L5, and then propagated past the site of TTX injection (Fig. 6A,C). However, up-state propagation was delayed relative to control by $\sim 50 \mathrm{~ms}$ in ROIs located $500 \mu \mathrm{m}$ from the TTX injection in L2/3 and L5A/B (Fig. 6C, delay measured at 5SDs, indicated by dashed red 


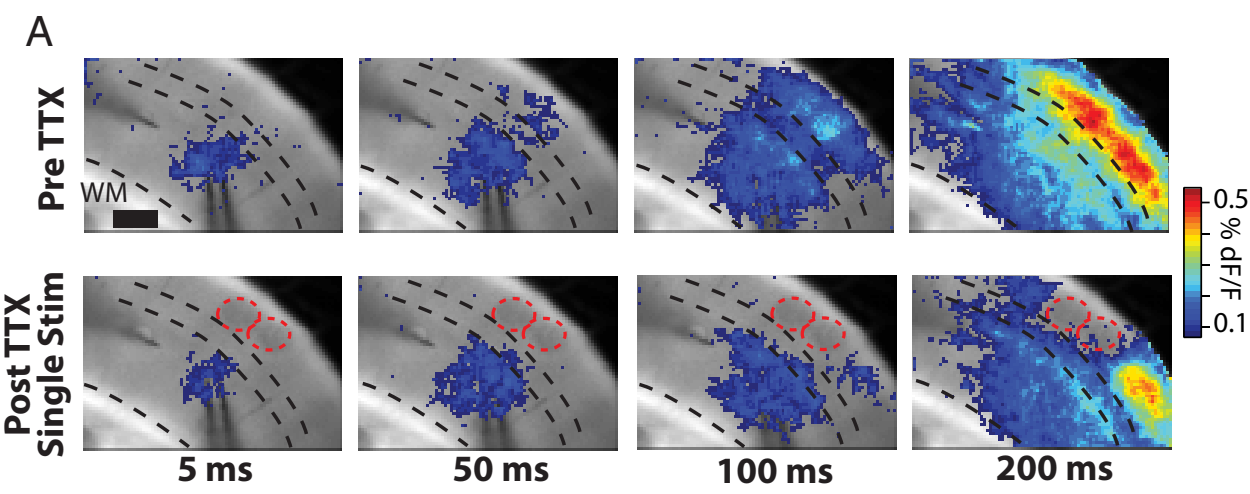

B
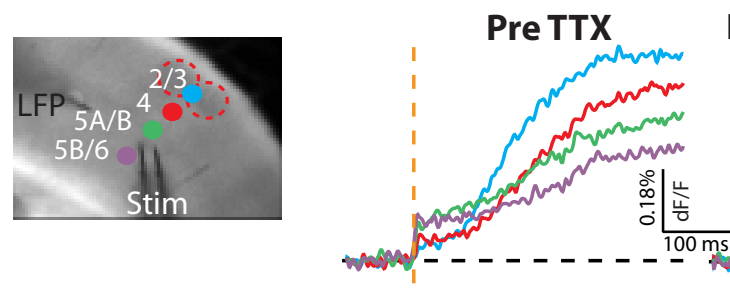

Post TTX - Single Stim

C

\section{ROIs adjacent to TTX}

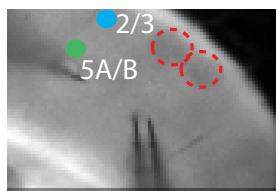

Pre TTX

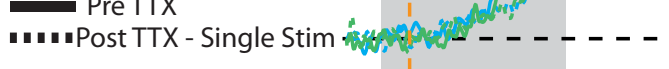

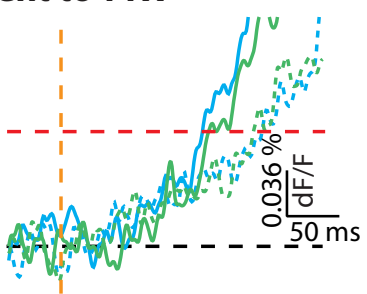

D

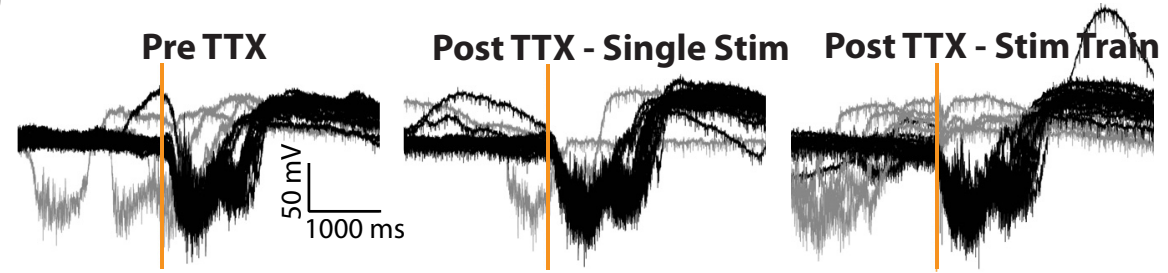

Figure 6. TTX injection in L2/3 does not block up-state initiation triggered by L5 stimulation. $A$, VSD movie frames before (top) and after (bottom) TTX injection into L2/3 (red dashed circles). Response shown is to a single $\mathrm{L} 5$ stimulus $(5 \mu \mathrm{A}, 100 \mu \mathrm{s})$, which triggers an up-state that propagates beyond the borders of the TTX injection and across the imaged area. Times are poststimulus. Scale bar, $500 \mu \mathrm{m} . \boldsymbol{B}$, ROls through the depth of the cortex aligned vertically to the stimulation site. Activity is sustained below the TTX injection for the duration of the imaging time ( $512 \mathrm{~ms}$ in this experiment) after a single $L 5$ stimulus. $C$, ROIs in L2/3 and L5A/B $500 \mu \mathrm{m}$ adjacent to the TTX injection site, and traces in response to a single $L 5$ stimulation. The up-state propagates to these ROIs after TTX injection, but is delayed by $\sim 50 \mathrm{~ms}$ relative to control conditions. Red dashed lines indicate 5 SDs above baseline noise. $\boldsymbol{D}$, LFP recording (see $A$ and $\boldsymbol{B}$ above for labeled position in reference image). Shown are individual trials aligned to the stimulation time (orange line). The black traces represent trials in which spontaneous up-states (gray) did not interfere with the stimulation; these trials were averaged in the VSD movies above. Note up-states triggered by the stimulation and also the occurrence of spontaneous up-states recorded after TTX injection.

line). For the population, up-state propagation was delayed by $49 \pm$ $33 \mathrm{~ms}$ in $\mathrm{L} 2 / 3$ and $52 \pm 23 \mathrm{~ms}$ in L5A/B $(n=4$ slices; $n=8$ ROIs for each layer). Finally, both triggered and spontaneous up-states were observed in the adjacent LFP after TTX injection (Fig. 6D).

These data reveal a key role for $\mathrm{L} 5$ in the initiation of up-states and also indicate that it is crucial for the propagation of excitation across the cortex. Furthermore, they reveal an apparent weakness in L2/3 horizontal connections, as even stimulus trains failed to propagate excitation to regions adjacent to the TTX injection column. We next investigated the effect of TTX injection in L2/3 and L5 on the propagation of ongoing up-states, triggered hundreds of micrometers away from the site of the injection. Under these conditions, physiological inputs rather than electrical stimulation provide excitatory drive in the column in which TTX is injected.
Injection of TTX into L2/3 does not stop up-state propagation We triggered up-states with VB stimulation and injected TTX into $\mathrm{L} 2 / 3$ adjacent to the primary column ( $n=13$ slices). A representative example is shown in Figure 7. TTX in L2/3 did not impede the propagation of the up-state, which traveled successfully through infragranular layers past the injection site and activated all layers (Fig. 7A). The activity that was eliminated by the TTX can be seen by subtracting the peak map (see Materials and Methods) of the response after TTX from that of the control response (Fig. $7 B$ ). The subtraction map reveals a zone of blocked activity that matches the area delimited by the dye and an additional surrounding area with a small but significant reduction of activity.

To quantify the effects of TTX, we examined four ROIs through the depth of the cortex as above (L2/3, L4, L5A/B, 
A

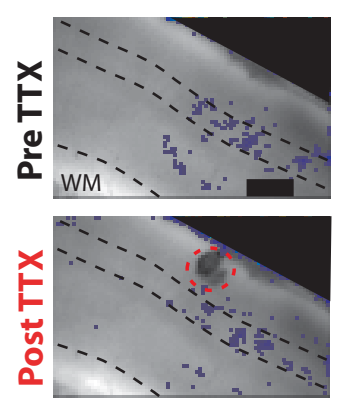

$7 \mathrm{~ms}$

B
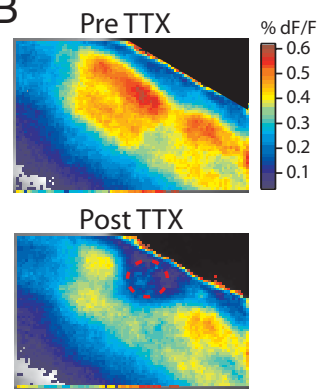

Subtraction

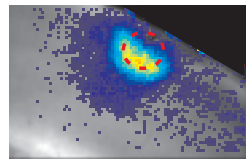

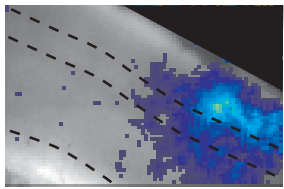

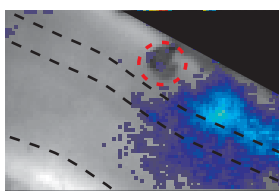

$25 \mathrm{~ms}$

C

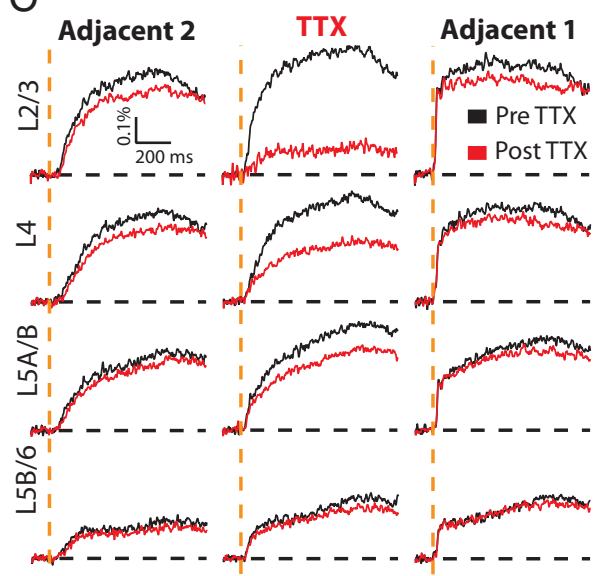

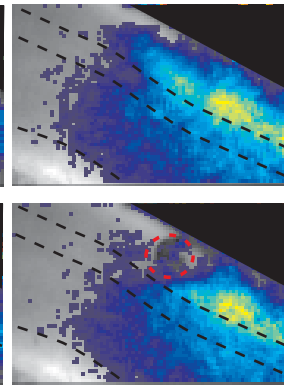

$75 \mathrm{~ms}$
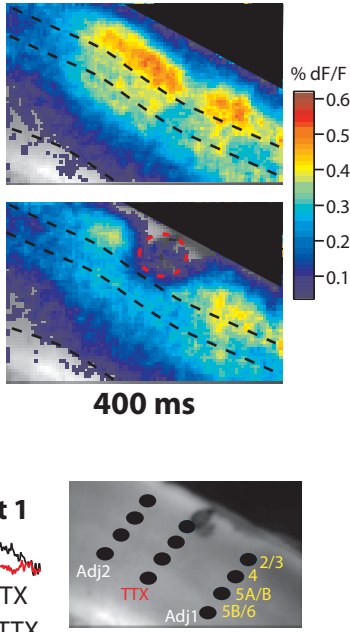

Adjacent 1 L2/3

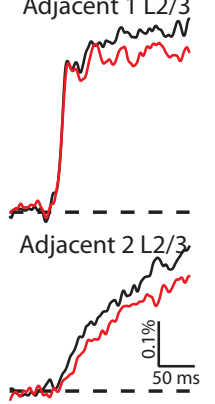

D

Layer 4 cortical stim
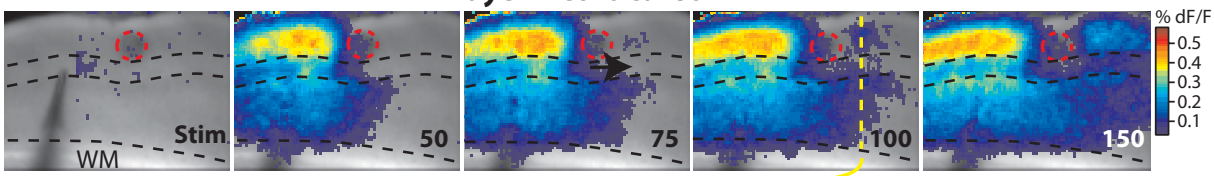

Second TTX puff
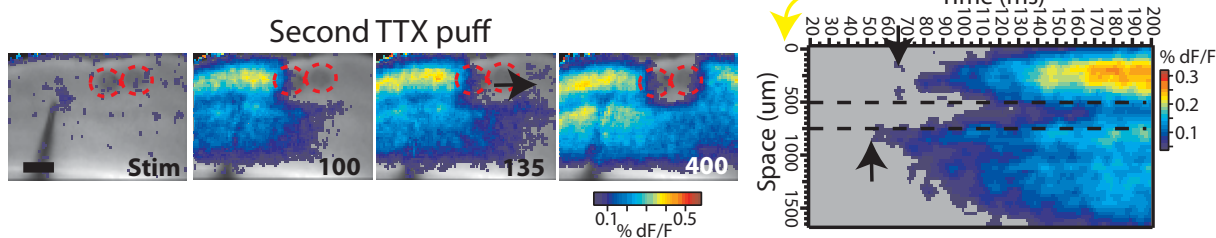

Figure 7. TTX injection into L2/3 does not stop up-state propagation and primarily affects the region around the puff and the layers immediately below. $\boldsymbol{A}$, VSD movie frames of a thalamocortically triggered up-state before and after TTX injection into L2/3. Coomassie blue added to the TTX solution produces a dark spot at the site of injection (red dashed circle). The up-state is initiated similarly in TTX and control conditions and then travels past the site of TTX via infragranular layers. Scale bar, $500 \mu \mathrm{m}$. Time is post-VB stimulation. $\boldsymbol{B}$, Peak maps of activity pre- and post-TTX and a subtraction image. The effect of TTX is largest centered at the site of the injection, with smaller changes in the $d F / F$ values immediately below and adjacent. $C$, ROls through the depth of the cortex in three columns before and after TTX injection in L2/3: TTX injection column, and two adjacent columns. A decrease in the dF/Famplitude after TTX is most prominent in the L2/3 ROI in the TTX column, with differences decreasing through the depth. Small decreases in the signal after TTX are also seen in the two adjacent columns in L2/3 and L4. At right are signals from ROIs in L2/3 close to the site of up-state initiation (top) and on the far side of the TTX injection (bottom). The up-state initiates with no delay after TTX, but a delay of $10 \mathrm{~ms}$ is observed on the far side of the injection. $\boldsymbol{D}$, Direct $L 4$ stimulation $(2 \mu \mathrm{A}, 100 \mu \mathrm{s})$. In the frame at 75 ms after stimulation, there is clear activation of $L 2 / 3$ from $L 5$ below. The arrow indicates that there is no $d F / F$ signal in $L 4$, which suggests that the signal in $L 2 / 3$ is not due to depolarization of $L 5$ apical dendrites but rather synaptic input to L2/3 from L5. This is confirmed in the line profile below (location of the line is indicated in the frame at $100 \mathrm{~ms}$; time axis is poststimulation; the arrows indicate appearance of activity in $\mathrm{L} 5$ and $\mathrm{L} 2 / 3$; the dashed lines bracket the borders of L4). A second injection of TTX next to the first, removing $1 \mathrm{~mm}$ of $\mathrm{L} 2 / 3$ horizontal connectivity, also failed to stop up-state propagation (bottom frames). Scale bar, $500 \mu \mathrm{m}$.

L5B/6). ROIs were obtained from the column where the TTX was injected ("TTX column") and from two adjacent columns $\sim 450-500 \mu \mathrm{m}$ on either side of the injection ("adjacent 1 " near the site of up-state initiation and "adjacent 2" on the far side of the TTX injection) (Fig. $7 C$, inset). The ROIs (Fig. $7 C$ ) demonstrated a large reduction in the $d F / F$ signal in L2/3 at the site of TTX injection, and a smaller effect on the
ROIs below. Small effects on the $d F / F$ signal amplitude were also found in the two adjacent columns. Furthermore, in adjacent 1 , the onset of the response and the rate of rise of the $d F / F$ signal were unaffected and therefore represent an intact initiation of the up-state, whereas on the far side of the TTX injection (adjacent 2), the up-state was delayed by $10 \mathrm{~ms}$ (Fig. 7C). 


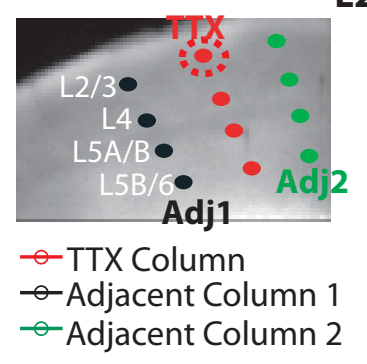

L2/3 TTX

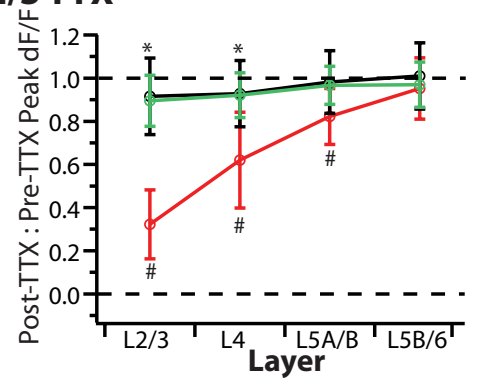

Figure 8. Quantification of the effect of $L 2 / 3$ TTX injection on up-state propagation. A schematic of ROl locations used for quantification is shown over a grayscale reference image (left). Plotted in each graph is the ratio of the peak dF/F signal after TTX to before TTX at each ROI (mean \pm SD) (see Materials and Methods). TTX in L2/3 ( $n=21$ slices; VB stim, $n=13 ; \mathrm{L} 4$ stim, $n=8$ ) primarily affects the column in which the injection occurred, and changes in $d F / F$ decrease through the depth of the cortex. Reduction in $d F / F$ signal in adjacent columns was small and limited to L2/3 and L4 ROIs. Student's $t$ test for difference from 1: ${ }^{*} p<0.05$, $\# p<0.001$.

We also triggered up-states in a more controlled manner with minimal cortical L4 stimulation ( $2-5 \mu \mathrm{A} ; n=9$ slices) (Fig. $7 D)$. The up-state always traveled past the TTX injection via infragranular layers, as with VB stimulation (Fig. 7D). This propagation was delayed with respect to control by $20-50 \mathrm{~ms}(n=6$ of 8 slices) or not delayed at all ( $n=2$ of 8 slices). Once it propagated under the site of TTX injection, the up-state clearly spread from L5A directly to L2/3, as $d F / F$ signal was only detectable in L4 after activation of $\mathrm{L} 2 / 3$ (Fig. $7 D$, frame at $100 \mathrm{~ms}$ ). This is also clearly visible in the line profile (Fig. $7 D$, dotted yellow line; arrows indicate sequential activation of L5A and L2/3), taken on the far side of the TTX injection. A second neighboring TTX injection in L2/3 also failed to stop up-state propagation via infragranular layers (Fig. 7D, "second TTX puff"). Although this example is from a thalamocortical connected slice, we recognized the potential, although not yet demonstrated in rat thalamocortical slices, for cortico-thalamo-cortical feedback loops, which would complicate interpretation of the results as a purely cortical phenomenon [see the studies by Theyel et al. (2010) and Golshani et al. (2001) for cortico-thalamo-cortical connectivity in mouse slices; see also Rigas and Castro-Alamancos (2007)]. Thus, as a further control, in the remainder of L4 stimulation experiments $(n=8)$, we used slices that were either immediately rostral or caudal to thalamocortical connected slices, and therefore not connected. The results from these experiments were not different from the example in Figure $7 D$ as well as experiments with VB stimulation, and thus the data were pooled.

From ROIs, we calculated the ratio of the peak $d F / F$ after TTX to before TTX to obtain the percentage maximum amplitude $d F / F$ reached relative to control (see Materials and Methods). Across the population (Fig. $8 ; n=21$ slices; VB stim, $n=13$; L4 stim, $n=8$ ), L2/3 TTX injection reduced the $d F / F$ signal around the injection site to $32 \pm 16 \%$ of the control peak amplitude. The difference between control and post-TTX $d F / F$ signals decreased as ROIs were analyzed in layers below, and was not statistically significantly different from 100\% for L5B/L6 (percentage of control peak, difference from 100\%: L4, $62 \pm 22 \%, p<0.001$; L $5 / B$, $82 \pm 13 \%, p<0.001 ; \mathrm{L} 5 \mathrm{~B} / 6,95 \pm 14 \%, p=0.13)$. In the columns $450-500 \mu \mathrm{m}$ adjacent to the site of TTX injection, the only statistically significant differences in $d F / F$ were measured in the L4 and L2/3 ROIs (L4 adjacent 1, $93 \pm 15 \%, p<0.05$; L4 adjacent 2, $92 \pm 10 \%, p<0.05 ; \mathrm{L} 2 / 3$ adjacent $1,92 \pm 18 \%, p<0.05 ; \mathrm{L} 2 / 3$ adjacent 2, $90 \pm 12 \%, p<0.05)$; however, these differences were small and most likely do not represent a reduction in the synaptic drive of the up-state. Importantly, once an up-state was initiated, it propagated past the site of TTX injection in all trials.

Thus, injection of TTX into a small (one to two barrel columns) region of L2/3 does not stop the spread of an up-state past the site of injection. We conclude that L2/3 is not critical for up-state propagation and has only a minimal impact on the recurrent activity of neighboring columns. We next injected TTX into L5 to determine the importance of infragranular layers for propagation.

\section{Injection of TTX into L5 stops up-state propagation and has a large effect on $\mathrm{L} 2 / 3$ above}

In stark contrast to the effect of injecting TTX into L2/3, a small injection of TTX into layer 5 (affecting L5A and at least part of L5B) could completely stop a traveling up-state (Fig. 9). In these experiments, we always initiated up-states with a L4 cortical stimulation because application of TTX in infragranular layers blocks afferent inputs from the thalamus en route to L4. In the representative example in Figure 9, TTX injection in L5 blocked the propagation of the up-state past the injection site in the majority of trials (13 of 18 trials), producing a vertical termination front spanning all layers of cortex ("no travel" trials; Fig. 9A, middle row). However, in 5 of 18 of the trials, the up-state propagated via layer $2 / 3$ past the injection site ("travel" trials; Fig. $9 A$, bottom row; $B$ ). Trials in which up-states propagated past the site of TTX injection occurred randomly among trials with failures and were not grouped at the beginning or end of a set of stimuli. Thus, there appears to be a threshold for recurrent excitation to generate an up-state in an all-or-none manner. Furthermore, projections within L2/3 are capable of reaching this threshold, but without input from L5 they often fail (see below and Discussion). The subtraction peak maps show the spatial extent of the effects of TTX in the travel and no-travel trials (Fig. 9C). In the travel trials, regions of the slice past the TTX injection clearly generate up-states that are similar to control.

We quantified $d F / F$ signals from ROIs as above for L2/3 TTX injections. In the column of ROIs between the stimulus and the TTX injection site (adjacent 1 ), the $d F / F$ signal was not different before and after TTX for both travel (pink traces) and no-travel trials (red traces) (Fig. 9D, adjacent 1), further demonstrating that TTX does not globally reduce excitability in the slice. There was no delay in the up-state onset and the rate of rise of the $d F / F$ signal was unaffected (Fig. 9D, left column, detail from L2/3 adjacent 1 ). In the TTX column, the $d F / F$ signal was greatly reduced through the depth of the cortex in both travel and no-travel trials (Fig. 9D, TTX). The reduction of $d F / F$ in $\mathrm{L} 2 / 3$ of the TTX column in travel trials suggests a driving influence of the L5 to L2/3 inputs (Shepherd and Svoboda, 2005; Shepherd et al., 2005; Bureau et al., 2006). In the column on the far side of the TTX injection (adjacent 2), the up-state was clearly absent in the no-travel trials, but it reached similar $d F / F$ amplitudes in the travel trials, albeit with a $70 \mathrm{~ms}$ delay (Fig. 9D, adjacent 2, see detail in left column, $\mathrm{L} 2 / 3$ adjacent 2 ). Thus, as with the injections in L2/3, up-states can be generated by local recurrent activity in the absence of long axonal activity.

For the population, injection of TTX into L5 ( $n=12$ slices) resulted in the block of propagating up-states in all trials in six slices and in at least one-half of trials in four slices. In the two remaining slices, the L5 TTX injection failed to block up-state propagation, which was successful via supragranular layers. We will first consider trials in which the up-state failed to propagate 
A
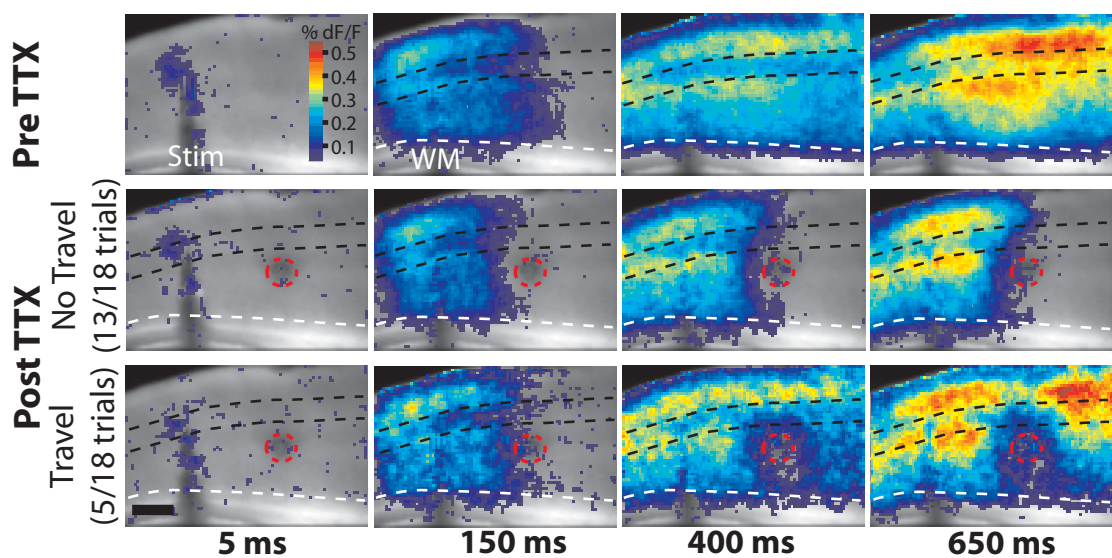

$400 \mathrm{~ms}$

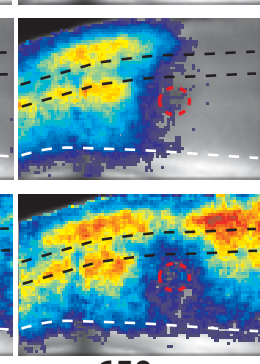

$650 \mathrm{~ms}$
B

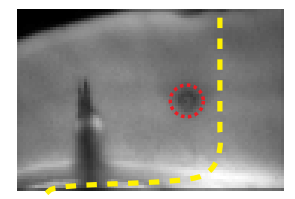

Time (ms)

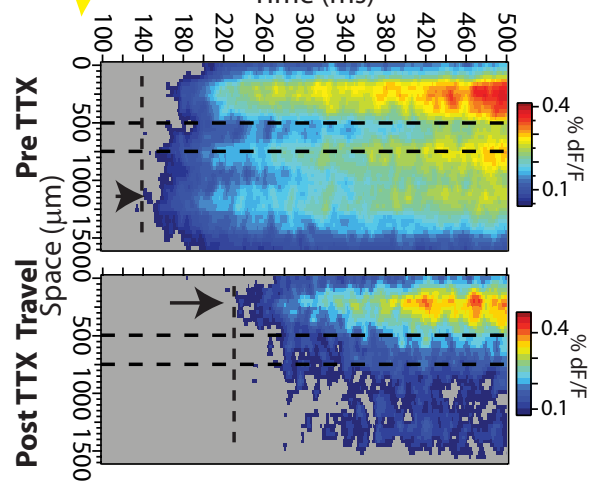

C

\section{Peak Map \\ Subtractions}

Wave Stops

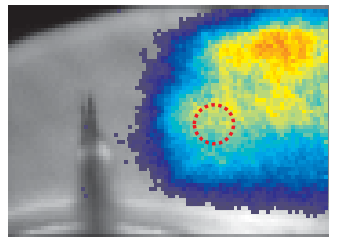

Wave Travels

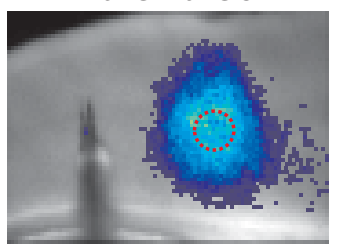

$0.1 \% \mathrm{dF} / \mathrm{F} \quad 0.6$
D
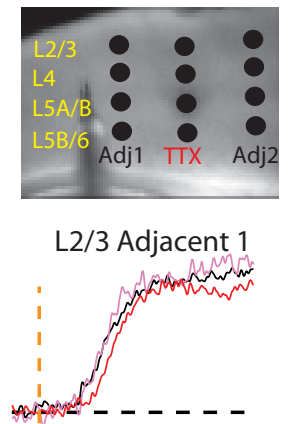

L2/3 Adjacent 2
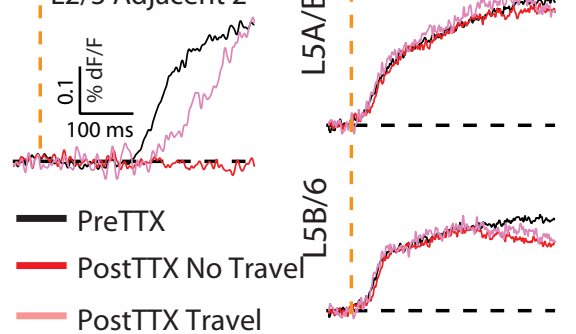

Adjacent 1
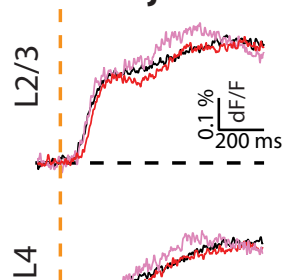

$\Xi$
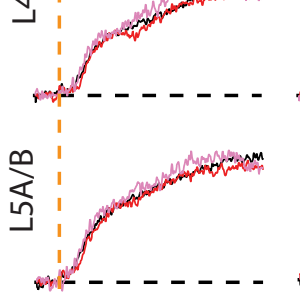
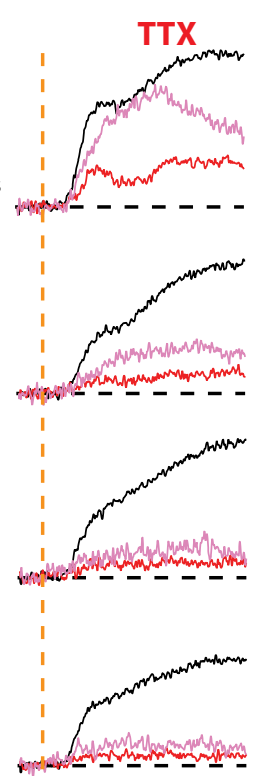

Adjacent 2
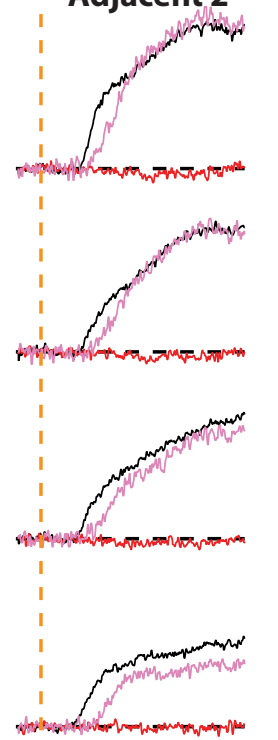

Figure 9. TTX injection in L5 can stop a propagating up-state. A, VSD movie frames. The top row is the average of trials pre-TTX. The middle row is the average of trials ( 13 of 18 ) in which an up-state failed to propagate past the site of TTX injection into L5 (no
(Fig. 10, no travel; $n=10$ slices). The $d F / F$ signal in the TTX injection column (red) was greatly decreased for all ROIs through the depth of the cortex (percentage of control peak, difference from $100 \%: \mathrm{L} 2 / 3$, $27 \pm 23 \%, p<0.001 ; \mathrm{L} 4,18 \pm 11 \%, p<$ $0.001 ; \mathrm{L} 5 \mathrm{~A} / \mathrm{B}, 15 \pm 6 \%, p<0.001 ; \mathrm{L} 5 \mathrm{~B} / 6$ $13 \pm 5 \%, p<0.001)$. In adjacent 1 (black), there was a small but significant decrease in the $d F / F$ signal through the depth (L2/3, $83 \pm 15 \%, p<0.01 ; \mathrm{L} 4,81 \pm$ $18, p<0.01 ; \mathrm{L} 5 \mathrm{~A} / \mathrm{B}, 80 \pm 14 \%, p<0.01$; L5B/6, $78 \pm 15 \%, p<0.01$ ). To demonstrate that there was not a global effect on excitably of the slice caused by the TTX injection, we also investigated an adjacent column further away $(>500 \mu \mathrm{m})$ (adjacent, gray). At these further distances, the $d F / F$ signals were not significantly different from control (L2/3, $93 \pm 16 \%, p=$ $0.2 ; \mathrm{L} 4,93 \pm 22 \%, p=0.36 ; \mathrm{L} 5 \mathrm{~A} / \mathrm{B}, 92 \pm$ $14 \%, p=0.12 ; \mathrm{L} 5 \mathrm{~B} / 6,91 \pm 13 \%, p=0.2$ ). Thus, even with the loss of up-states across most of the slice, local recurrent activity far enough away from the TTX injection could generate up-states similar to control.

For trials in which the up-state traveled past the TTX injection (Fig. 10, travel; $n=$ 6 slices), in the TTX column all but the

$\leftarrow$

travel). The bottom row is the average of trials (5 of 18) in which the up-state propagation succeeded (travel). Although the baseline noise is higher for travel trials (bottom row), the same color scale was used to highlight that a similar peak in $d F / F$ signal is reached on the far side of the TTX injection. Note, however, that this allows some noise peaks in the pseudocolor at baseline. Red dashed circle, TTX injection site. Scale bar, 500 $\mu \mathrm{m}$. $\boldsymbol{B}$, Line profile immediately adjacent to the site of TTX injection in $L 5$ (dashed red circle). Before TTX injection, the up-state propagates via infragranular layers (top profile). After TTX, in travel trials ( $n=5$ of 18 ), up-state propagation occurs via supragranular layers (bottom profile). The dashed vertical lines mark the first appearance of activity reaching 2 SDs above baseline noise along the line profile. The arrows highlight the layer in which this activity first appeared. The dashed horizontal lines bracket L4. C, Subtractions of peak maps relative to control for the travel and no-travel trials to show region of the slice affected by TTX injection. $\boldsymbol{D}$, ROIs through the depth of the cortex in three columns before and after TTX injection in L5: TTX injection column and two adjacent columns. In the TTX injection column, the dF/F signal is largely reduced in both travel and no-travel trials relative to control for all layers. Note, however, that the peak amplitude reached in the travel trials is greater than that of the no-travel trials, particularly in L2/3. In trials in which the up-state always travels, the $d F / F$ signal in the adjacent columns reaches the same peak amplitude as control, although with a delay of $\sim 70$ ms on the far side of the TTX. In trials in which the upstate fails to travel past the TTX injection, the $d F / F$ signal reaches similar but slightly smaller peak amplitudes in the adjacent column near the stimulation site, and is zero in the adjacent column on the far side of the TTX. The adjacent column ROIs from L2/3 are shown with an expanded timescale at left. 
L5 TTX

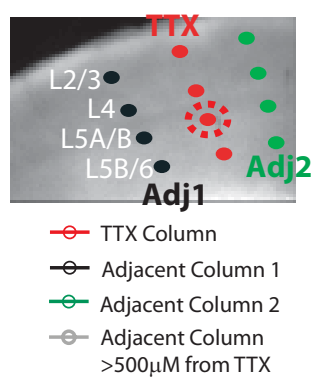

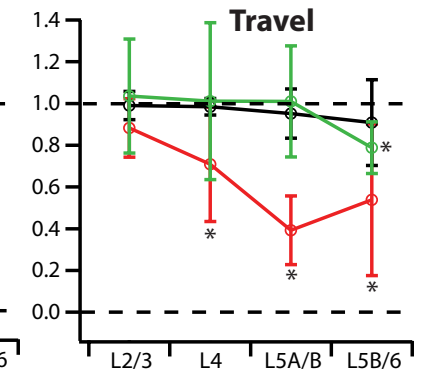

of the $V_{\mathrm{m}}$ depolarization during an upstate and calculated the ratio between post- and pre-TTX conditions (Fig. 11B) (see Materials and Methods). In experiments with L2/3 TTX injections, intracellular recordings in L5 ( $n=5$ cells) of the TTX column did not reveal significant differences from control at the level of the soma (percentage of control, mean \pm SD: $95 \pm 8 \%$; $p<0.05)$. However, in recordings made in L2/3 above a L5 TTX injection ( $n=4$ cells), significant reductions in the width at half-height were measured. In two cells, trials in which an up-state failed to propagate past the L5 TTX injection resulted in reductions in width at halfheight of 22 and $60 \%$ of control. Importantly, a similar reduction from control was measured for trials in which up-states propagated past the TTX injection (percentage of control: $51 \pm 19 \%$; $p<0.05$; $n=4$ cells). This is true even in the rare

L2/3 ROI was significantly different from the control peak (L2/3, $88 \pm 14 \%, p=0.1 ; \mathrm{L} 4,71 \pm 27 \%, p<0.05 ; \mathrm{L} 5 \mathrm{~A} / \mathrm{B}, 39 \pm 16 \%$, $p<0.01 ; \mathrm{L} 5 \mathrm{~B} / 6,54 \pm 36 \%, p<0.05)$. In the two columns $450-500 \mu \mathrm{m}$ adjacent to the TTX injection (adjacent 1, black, and adjacent 2, green), only the L5B/6 ROI in adjacent 2 was significantly different from control (adjacent 1: L2/3, $99 \pm 7 \%$, $p=0.74 ; \mathrm{L} 4,98 \pm 4, p=0.36 ; \mathrm{L} 5 \mathrm{~A} / \mathrm{B}, 95 \pm 12 \%, p=0.36 ; \mathrm{L} 5 \mathrm{~B} / 6$, $91 \pm 21 \%, p=0.37$. adjacent $2: \mathrm{L} 2 / 3,104 \pm 27 \%, p=0.76 ; \mathrm{L} 4$, $101 \pm 38, p=0.95 ; \mathrm{L} 5 \mathrm{~A} / \mathrm{B}, 101 \pm 27 \%, p=0.93 ; \mathrm{L} 5 \mathrm{~B} / 6,79 \pm$ $12 \%, p<0.05)$.

We draw two conclusions from these data. First, a peak $d F / F$ signal (and thus depolarization) relative to control must be reached in L2/3 for an up-state to propagate via this layer. This represents the threshold for recurrent excitation necessary to produce an up-state. Second, once an up-state travels past the TTX injection, vertical connectivity facilitates up-state propagation.

\section{L5 excitatory input to $\mathrm{L} 2 / 3$ increases the probability of reaching up-state threshold}

To better understand the effect of TTX within a column, we performed experiments with combined VSD and intracellular recordings. In the example in Figure $11 \mathrm{~A}$ (same slice from Fig. 9), a small ROI (4 pixels $=\sim 130 \mu \mathrm{m}$ diameter) was taken in the vicinity of an intracellular recording in L2/3 (Fig. $11 \mathrm{~A}$, inset). TTX was injected into L5 and trials were segregated as above (i.e., pre-TTX, post-TTX travel trials, post-TTX no-travel trials) for averaging purposes. After L5 TTX, the time course and change in amplitude of the $V_{\mathrm{m}}$ recording (continuous traces) matched the $d F / F$ signal from the ROI (dashed traces). Importantly, the peak $V_{\mathrm{m}}$ depolarization of the cell and the corresponding $d F / F$ signal were similar to the control conditions in travel trials (Fig. $11 \mathrm{~A}$, right column). Thus, up-state propagation past a TTX injection in L5 requires sufficient depolarization of L2/3. However, because up-states failed to propagate in the majority of trials, we conclude that L5 input to L2/3 provides depolarization that increases the probability that up-state threshold will be reached.

In contrast, up-state threshold in L5 is not dependent on input from L2/3, as demonstrated by consistent up-state propagation after TTX injection in L2/3. To further quantify changes in upstates after TTX injection, we measured the width at half-height experiment in which an up-state propagated in all trials (Fig. $11 C$ ). Thus, in the absence of $\mathrm{L} 5$, the peak amplitude reached in L2/3 during traveling up-states is not different from control; however, the synaptic drive and duration of the upstate is significantly reduced. This result indicates that, for an up-state to propagate, there is a threshold of peak activation that must be reached, and that L5 input makes this threshold more likely to be reached in $\mathrm{L} 2 / 3$.

\section{Control for slice angle}

Finally, because slices were cut in the thalamocortical plane (see Materials and Methods), we repeated the experiments in coronal slices as a control for slice angle. Results were identical for both L2/3 TTX injections $(n=2)$ and L5 TTX injections $(n=2)$, and were pooled with the rest of the data.

\section{Discussion}

Local circuits in neocortex are connected across layers and columns via feedforward and feedback pathways (Thomson and Bannister, 2003; Shepherd et al., 2005; Douglas and Martin, 2007). The details of laminar connectivity are based on recordings from pairs of neurons (Feldmeyer et al., 2002; Thomson et al., 2002) and from circuit mapping based on glutamate uncaging (Shepherd et al., 2005; Schubert et al., 2007). Here, we show that local recurrent network activity triggered by thalamic input preferentially propagates within a column from $\mathrm{L} 4 \rightarrow \mathrm{L} 2 / 3$ and then to deep layers. However, we demonstrate that $\mathrm{L} 5$ amplifies activity in L2/3 and is necessary for the spread of recurrent excitation across cortical columns.

\section{Up-states in vivo and in vitro share similar characteristics}

Originally described in vivo in cats under anesthesia and during slow-wave sleep (Steriade et al., 1993c), spontaneous cortical upand down-states occur in vitro under ionic conditions mimicking those observed in situ (Sanchez-Vives and McCormick, 2000). Both in vivo and in slices, spontaneous up-states are initiated primarily in deep layers (Sanchez-Vives and McCormick, 2000; Sakata and Harris, 2009). However, in agreement with our data, sensory triggered up-states start in thalamo-recipient layers (Sakata and Harris, 2009). Furthermore, single whisker deflections produce propagating waves imaged with VSDs (Civillico 
and Contreras, 2006; Ferezou et al., 2006) similar to our observations with single thalamic stimuli. Although corticothalamo-cortical loops shape the frequency components of up-states in vivo, up-states are cortically generated and persist even after extensive thalamic lesions (Steriade et al., 1993b). Thus, we conclude that our findings are applicable to the connectivity and behavior of cortical circuits in the intact animal.

\section{Interaction of local recurrent circuits within a cortical column}

Although we observed monosynaptic VB input in both L4 and L5B/6 [in agreement with studies by Agmon and Connors (1992), Laaris et al. (2000), de Kock et al. (2007), Meyer et al. (2010a,b), and Wimmer et al. (2010)], L5B/6 activity faded while that in L4 persisted and after a delay invaded L2/3. Thus, persistent activity within a column was observed to preferentially follow a L4 $\rightarrow$ L2/3 $\rightarrow$ L5 temporal sequence. Recurrent excitation amplifies spiking in local networks (Compte et al., 2003) and has been proposed to amplify afferent input to L4 (Douglas et al., 1995). L4 provides strong feedforward input to L2/3 (Lübke et al., 2003; Shepherd and Svoboda, 2005), and the probability that two $\mathrm{L} 2 / 3$ neurons are connected is higher when they share a common input from L4 (Yoshimura et al., 2005). Thus, VB input generates recurrent excitation in L4, which is further amplified in $\mathrm{L} 2 / 3$, leading to strong activation of L5 (Thomson and Deuchars, 1997). The inputs to and outputs from L6 in rodent barrel cortex are weak relative to connectivity in the layers above (Shepherd and Svoboda, 2005; Hooks et al., 2011). Furthermore, although $\mathrm{L} 5 \mathrm{~B}$ receives input from L6 (Schubert et al., 2001), there is not a prominent ascending projection from L5B (Shepherd and Svoboda, 2005; Schubert et al., 2007). Thus, inputs to L5B/6 would not be expected to engage the column. L5A, however, does project to L2/3 (Shepherd and Svoboda, 2005; Shepherd et al., 2005), and our data show that this ascending projection provides a strong driving input to L2/3 within a column, generating a powerful feedback loop among these layers.

\section{Propagation of up-states to neighboring cortical columns}

Our data revealed that L5 horizontal projections are consistently effective at propagating up-states to a neighboring column, whereas L2/3 horizontal projections, without the additional excitatory drive provided by L5A, are very ineffective.

L5A receives much of its excitatory input from L5A itself, both within a column and from neighboring columns (Schubert et al., 2006; Frick et al., 2008). We observe L5A being activated before deeper layers as an up-state propagates (Fig. 7D), indicating that
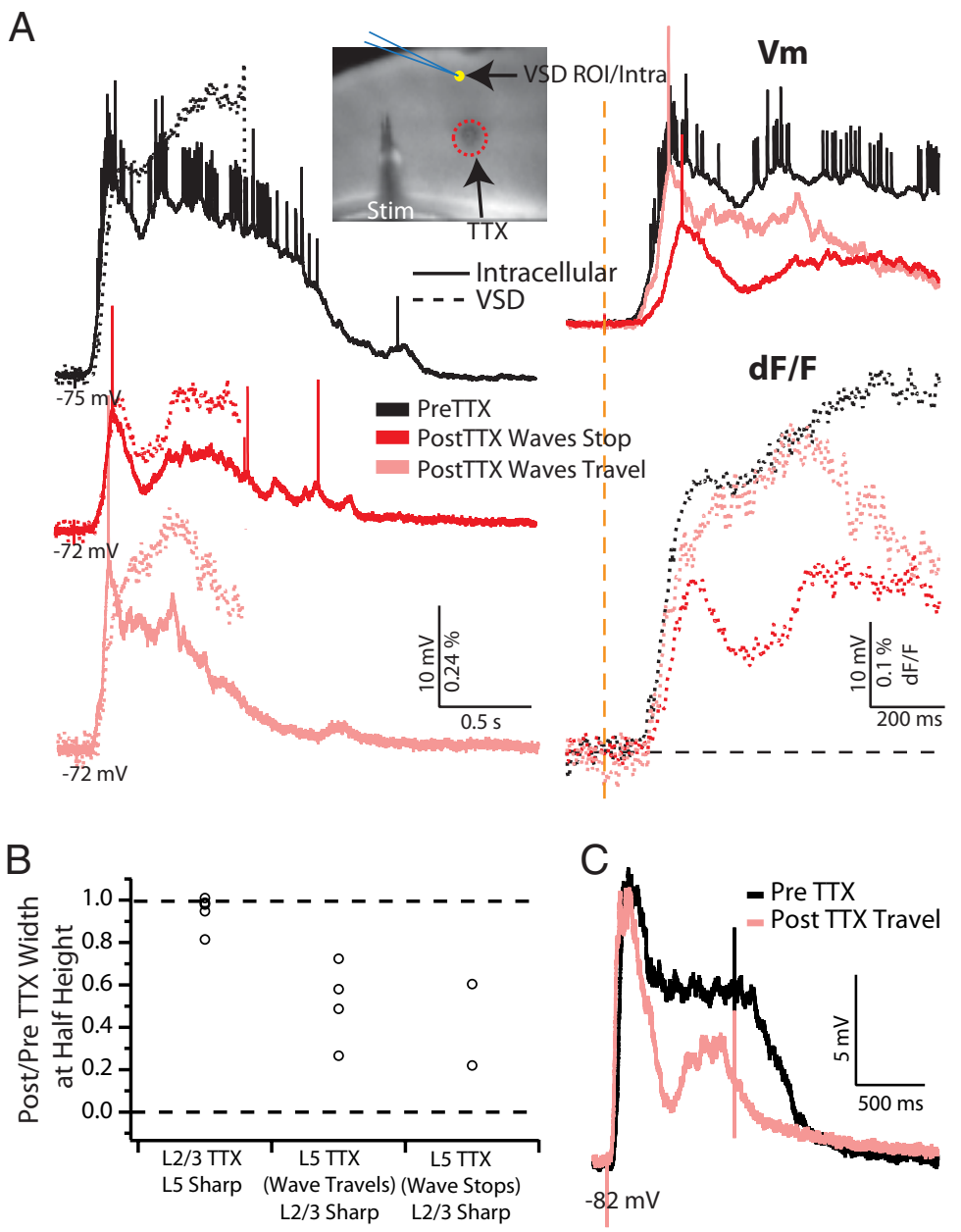

Figure 11. Intracellular recordings in $\mathrm{L} 2 / 3$ of the TTX column demonstrate strong ascending $\mathrm{L} 5$ input. $A$, Simultaneous VSD and intracellular recordings from the experiment in Figure 9. A small ROI (yellow circle) was taken in the region of the intracellular width at half-height of up-states measured from intracellular recordings post-TTX to pre-TTX. TTX injection in L2/3 does not reduce (he same column of the injection ( $n=5$ cells). However, TTX injection in L5 does

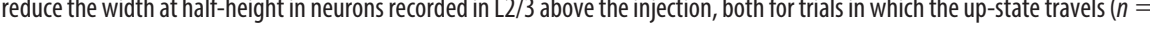
ells) and trials in which the wave fails to travel ( $n=2$ cells) past the TTX. Each circle represents data from a single neuron. $C$ 作 site of TTX injection in all trials. Note that the peak amplitude of the initial phase of the up-state is the same as control; however, depolarization is not sustained relative to control. All traces are averages of multiple trials.

this transcolumnar communication is particularly strong. Thicktufted pyramidal cells in L5B also project within L5 to neighboring columns (Schubert et al., 2001; Larsen et al., 2007; Oberlaender et al., 2011). These cells are intrinsically bursting (IB) (Chagnac-Amitai and Connors, 1989; Hefti and Smith, 2000; Schubert et al., 2001), generating spike bursts of three to five action potentials at $200 \mathrm{~Hz}$ (Connors et al., 1982; Contreras, 2004), which may boost connection strength. IB cells are also found within L5A (Schubert et al., 2006). Finally, L5A projects to L5B (Schubert et al., 2001), which could further drive activity throughout the entire layer.

Although L2/3 cells also project within supragranular layers (Feldmeyer et al., 2006), properties of these connections may limit their ability to propagate up-states. One constraint is the drop of up to 10 -fold in connection probability among L2/3 cells over distances of 20-150 $\mu \mathrm{m}$, observed in visual, somatosensory, and auditory cortex (Holmgren et al., 2003; Oswald and Reyes, 2008). Furthermore, neighboring L $2 / 3$ neurons are more weakly connected than L5 neurons, with maximum monosynaptic EP- 
SPs in L2/3 of $3 \mathrm{mV}$ compared with $6 \mathrm{mV}$ or greater in L5 (Markram et al., 1997; Thomson and Deuchars, 1997; Feldmeyer et al., 2006; Frick et al., 2008). Finally, all pyramidal cells in L2/3 are regular spiking (RS); IB cells are found mainly in deeper layers (Chagnac-Amitai and Connors, 1989).

Differences in inhibition and resting $V_{\mathrm{m}}$ may also influence network excitability. In L2/3, pyramidal cells were found to receive 10 times as many inhibitory connections as excitatory connections, many of which were reciprocal (Holmgren et al., 2003). In L5, although RS cells receive inhibition from multiple sources, IB cells receive relatively few inhibitory inputs (Schubert et al., 2001). Furthermore, L2/3 neurons are more hyperpolarized at rest than L5 neurons (Manns et al., 2004; Lefort et al., 2009), such that L5 cells even fire spontaneously at rest in between up-states (Sanchez-Vives and McCormick, 2000).

Our results show that when L2/3 is blocked with TTX, upstates propagate from L5 to L2/3 on the far side of the TTX injection. Projections from L5 $\rightarrow \mathrm{L} 2 / 3$ have been described in somatosensory, visual, and motor cortices (Yuste et al., 1997; Shepherd and Svoboda, 2005; Shepherd et al., 2005; Yoshimura et al., 2005; Bureau et al., 2006; Larsen et al., 2007; Weiler et al., 2008). In the barrel cortex, L5A provides a particularly strong ascending projection (Shepherd and Svoboda, 2005; Shepherd et al., 2005; Bureau et al., 2006), and our data show that this sublayer may play a primary role in both initiating and sustaining activity in L2/3 within the same column (Figs. $5 C, 7$ ).

We propose the following model for up-state propagation. Strong horizontal projections within L5, most likely primarily L5A, excite neighboring local circuits. Input from L5A $\rightarrow \mathrm{L} 2 / 3$ is necessary to overcome the hyperpolarization, inhibition, and sparse connectivity of subnetworks in superficial layers to increase the probability that $\mathrm{L} 2 / 3$ reaches threshold for producing an up-state. Once an up-state is initiated in L2/3, feedback projections from L2/3 $\rightarrow$ L5 help to drive activity in deeper layers, which then spreads to the next column.

\section{Relationship to models of epilepsy}

Paroxysmal activity most likely propagates via the same pathways as described here for up-states. Indeed, a popular model for studying epilepsy that consists of blocking GABA receptors to unmask excitatory circuits, results in similar conclusions. Epileptiform events start spontaneously in deeper layers (Connors, 1984), are all-or-none events (Gutnick et al., 1982), spread as a vertical wave-front recruiting L2/3 and L5 (Chervin et al., 1988; Pinto et al., 2005), but require L5 for propagation (Silva et al., 1991; Telfeian and Connors, 1998; Pinto et al., 2005). Indeed, VSD imaging during GABA block reveals a similar pattern of activity initiation and propagation observed in the present study (Laaris et al., 2000). Up-states under our conditions differ from these paroxysmal events in terms of depolarization amplitude [up-state, $10-20 \mathrm{mV}$, vs seizure, $20-50 \mathrm{mV}$ (Gutnick et al., 1982)], lack of sodium spike inactivation, propagation velocity [20 vs $60-90 \mathrm{~mm} / \mathrm{s}$ (Chervin et al., 1988; Pinto et al., 2005)], and the participation of inhibition, which is at least partially blocked in those cited experiments. Up-states themselves are not epileptiform events and consist of balanced excitation and inhibition shown with intracellular recordings both in vivo and in vitro (Shu et al., 2003; Haider et al., 2006). However, the convergence of our results using up-states and these previous studies of seizure activity is striking. Furthering this connection, during slow-wave sleep in vivo, up-states can transition to seizures (Steriade and Contreras, 1995; Steriade et al., 1998).

\section{Functional implications}

Regardless of network state, whisker deflections result in large, multibarrel subthreshold receptive fields through all layers (Brecht and Sakmann, 2002; Brecht et al., 2003; Manns et al., 2004; Roy et al., 2011). VSD imaging of L2/3 in vivo demonstrated initial activation of a single barrel, and subsequent spread of depolarization across the barrel field (Petersen et al., 2003; Civillico and Contreras, 2006; Ferezou et al., 2006), similar to our observations in slices. In vivo, spiking in $\mathrm{L} 2 / 3$ is sparse, with only $3-10 \%$ of $\mathrm{L} 2 / 3$ neurons firing action potentials in response to whisker reflection (Brecht et al., 2003; Crochet et al., 2011). Thus, it seems unlikely that the output of $\mathrm{L} 2 / 3$ is driving the spread of excitation across the cortex. We propose that L5 is primarily responsible for this distribution of excitation across neighboring columns, including depolarization observed in L2/3. Furthermore, given the delay in L5 activation upon block of overlying $\mathrm{L} 2 / 3$, we propose that spatiotemporal patterns of cortical activity observed in L2/3 in response to whisker deflection are due to the interaction between supragranular and infragranular layers.

\section{References}

Agmon A, Connors BW (1991) Thalamocortical responses of mouse somatosensory (barrel) cortex in vitro. Neuroscience 41:365-379.

Agmon A, Connors BW (1992) Correlation between intrinsic firing patterns and thalamocortical synaptic responses of neurons in mouse barrel cortex. J Neurosci 12:319-329.

Amzica F, Steriade M (1995) Short- and long-range neuronal synchronization of the slow $(<1 \mathrm{~Hz})$ cortical oscillation. J Neurophysiol 73:20-38.

Brecht M, Sakmann B (2002) Dynamic representation of whisker deflection by synaptic potentials in spiny stellate and pyramidal cells in the barrels and septa of layer 4 rat somatosensory cortex. J Physiol 543:49-70.

Brecht M, Roth A, Sakmann B (2003) Dynamic receptive fields of reconstructed pyramidal cells in layers 3 and 2 of rat somatosensory barrel cortex. J Physiol 553:243-265.

Bureau I, von Saint Paul F, Svoboda K (2006) Interdigitated paralemniscal and lemniscal pathways in the mouse barrel cortex. PLoS Biol 4:e382.

Chagnac-Amitai Y, Connors BW (1989) Synchronized excitation and inhibition driven by intrinsically bursting neurons in neocortex. J Neurophysiol 62:1149-1162.

Chervin RD, Pierce PA, Connors BW (1988) Periodicity and directionality in the propagation of epileptiform discharges across neocortex. J Neurophysiol 60:1695-1713.

Civillico EF, Contreras D (2005) Comparison of responses to electrical stimulation and whisker deflection using two different voltage-sensitive dyes in mouse barrel cortex in vivo. J Membr Biol 208:171-182.

Civillico EF, Contreras D (2006) Integration of evoked responses in supragranular cortex studied with optical recordings in vivo. J Neurophysiol 96:336-351.

Cohen LB, Salzberg BM (1978) Optical measurement of membrane potential. Rev Physiol Biochem Pharmacol 83:35-88.

Compte A, Sanchez-Vives MV, McCormick DA, Wang XJ (2003) Cellular and network mechanisms of slow oscillatory activity $(<1 \mathrm{~Hz})$ and wave propagations in a cortical network model. J Neurophysiol 89:2707-2725.

Connors BW (1984) Initiation of synchronized neuronal bursting in neocortex. Nature 310:685-687.

Connors BW, Gutnick MJ, Prince DA (1982) Electrophysiological properties of neocortical neurons in vitro. J Neurophysiol 48:1302-1320.

Contreras D (2004) Electrophysiological classes of neocortical neurons. Neural Netw 17:633-646.

Contreras D, Llinás R (2001) Voltage-sensitive dye imaging of neocortical spatiotemporal dynamics to afferent activation frequency. J Neurosci 21:9403-9413.

Crochet S, Poulet JF, Kremer Y, Petersen CC (2011) Synaptic mechanisms underlying sparse coding of active touch. Neuron 69:1160-1175.

de Kock CP, Bruno RM, Spors H, Sakmann B (2007) Layer- and cell-typespecific suprathreshold stimulus representation in rat primary somatosensory cortex. J Physiol 581:139-154.

Douglas RJ, Martin KA (2004) Neuronal circuits of the neocortex. Annu Rev Neurosci 27:419-451. 
Douglas RJ, Martin KA (2007) Mapping the matrix: the ways of neocortex. Neuron 56:226-238.

Douglas RJ, Koch C, Mahowald M, Martin KA, Suarez HH (1995) Recurrent excitation in neocortical circuits. Science 269:981-985.

Feldmeyer D, Lübke J, Silver RA, Sakmann B (2002) Synaptic connections between layer 4 spiny neurone-layer $2 / 3$ pyramidal cell pairs in juvenile rat barrel cortex: physiology and anatomy of interlaminar signalling within a cortical column. J Physiol 538:803-822.

Feldmeyer D, Lübke J, Sakmann B (2006) Efficacy and connectivity of intracolumnar pairs of layer $2 / 3$ pyramidal cells in the barrel cortex of juvenile rats. J Physiol 575:583-602.

Ferezou I, Bolea S, Petersen CC (2006) Visualizing the cortical representation of whisker touch: voltage-sensitive dye imaging in freely moving mice. Neuron 50:617-629.

Frick A, Feldmeyer D, Helmstaedter M, Sakmann B (2008) Monosynaptic connections between pairs of L5A pyramidal neurons in columns of juvenile rat somatosensory cortex. Cereb Cortex 18:397-406.

Golshani P, Liu XB, Jones EG (2001) Differences in quantal amplitude reflect GluR4-subunit number at corticothalamic synapses on two populations of thalamic neurons. Proc Natl Acad Sci U S A 98:4172-4177.

Gutnick MJ, Connors BW, Prince DA (1982) Mechanisms of neocortical epileptogenesis in vitro. J Neurophysiol 48:1321-1335.

Haider B, Duque A, Hasenstaub AR, McCormick DA (2006) Neocortical network activity in vivo is generated through a dynamic balance of excitation and inhibition. J Neurosci 26:4535-4545.

Hefti BJ, Smith PH (2000) Anatomy, physiology, and synaptic responses of rat layer $\mathrm{V}$ auditory cortical cells and effects of intracellular $\mathrm{GABA}_{\mathrm{A}}$ blockade. J Neurophysiol 83:2626-2638.

Holmgren C, Harkany T, Svennenfors B, Zilberter Y (2003) Pyramidal cell communication within local networks in layer $2 / 3$ of rat neocortex. J Physiol 551:139-153.

Hooks BM, Hires SA, Zhang YX, Huber D, Petreanu L, Svoboda K, Shepherd GM (2011) Laminar analysis of excitatory local circuits in vibrissal motor and sensory cortical areas. PLoS Biol 9:e1000572.

Laaris N, Carlson GC, Keller A (2000) Thalamic-evoked synaptic interactions in barrel cortex revealed by optical imaging. J Neurosci 20:1529-1537.

Land PW, Kandler K (2002) Somatotopic organization of rat thalamocortical slices. J Neurosci Methods 119:15-21.

Larsen DD, Wickersham IR, Callaway EM (2007) Retrograde tracing with recombinant rabies virus reveals correlations between projection targets and dendritic architecture in layer 5 of mouse barrel cortex. Front Neural Circuits 1:5.

Lefort S, Tomm C, Floyd Sarria JC, Petersen CC (2009) The excitatory neuronal network of the $\mathrm{C} 2$ barrel column in mouse primary somatosensory cortex. Neuron 61:301-316.

Lübke J, Egger V, Sakmann B, Feldmeyer D (2000) Columnar organization of dendrites and axons of single and synaptically coupled excitatory spiny neurons in layer 4 of the rat barrel cortex. J Neurosci 20:5300-5311.

Lübke J, Roth A, Feldmeyer D, Sakmann B (2003) Morphometric analysis of the columnar innervation domain of neurons connecting layer 4 and layer 2/3 of juvenile rat barrel cortex. Cereb Cortex 13:1051-1063.

Manns ID, Sakmann B, Brecht M (2004) Sub- and suprathreshold receptive field properties of pyramidal neurones in layers $5 \mathrm{~A}$ and $5 \mathrm{~B}$ of rat somatosensory barrel cortex. J Physiol 556:601-622.

Markram H, Lübke J, Frotscher M, Roth A, Sakmann B (1997) Physiology and anatomy of synaptic connections between thick tufted pyramidal neurones in the developing rat neocortex. J Physiol 500:409-440.

Meyer HS, Wimmer VC, Oberlaender M, de Kock CP, Sakmann B, Helmstaedter M (2010a) Number and laminar distribution of neurons in a thalamocortical projection column of rat vibrissal cortex. Cereb Cortex 20:2277-2286.

Meyer HS, Wimmer VC, Hemberger M, Bruno RM, de Kock CP, Frick A, Sakmann B, Helmstaedter M (2010b) Cell type-specific thalamic innervation in a column of rat vibrissal cortex. Cereb Cortex 20:2287-2303.

Morishima M, Kawaguchi Y (2006) Recurrent connection patterns of corticostriatal pyramidal cells in frontal cortex. J Neurosci 26:4394-4405.

Obaid AL, Loew LM, Wuskell JP, Salzberg BM (2004) Novel naphthylstyrylpyridium potentiometric dyes offer advantages for neural network analysis. J Neurosci Methods 134:179-190.
Oberlaender M, Boudewijns ZS, Kleele T, Mansvelder HD, Sakmann B, de Kock CP (2011) Three-dimensional axon morphologies of individual layer 5 neurons indicate cell type-specific intracortical pathways for whisker motion and touch. Proc Natl Acad Sci U S A 108:4188-4193.

Oswald AM, Reyes AD (2008) Maturation of intrinsic and synaptic properties of layer $2 / 3$ pyramidal neurons in mouse auditory cortex. J Neurophysiol 99:2998-3008.

Petersen CC, Sakmann B (2001) Functionally independent columns of rat somatosensory barrel cortex revealed with voltage-sensitive dye imaging. J Neurosci 21:8435-8446.

Petersen CC, Grinvald A, Sakmann B (2003) Spatiotemporal dynamics of sensory responses in layer $2 / 3$ of rat barrel cortex measured in vivo by voltage-sensitive dye imaging combined with whole-cell voltage recordings and neuron reconstructions. J Neurosci 23:1298-1309.

Pinto DJ, Hartings JA, Brumberg JC, Simons DJ (2003) Cortical damping: analysis of thalamocortical response transformations in rodent barrel cortex. Cereb Cortex 13:33-44.

Pinto DJ, Patrick SL, Huang WC, Connors BW (2005) Initiation, propagation, and termination of epileptiform activity in rodent neocortex in vitro involve distinct mechanisms. J Neurosci 25:8131-8140.

Rigas P, Castro-Alamancos MA (2007) Thalamocortical Up states: differential effects of intrinsic and extrinsic cortical inputs on persistent activity. J Neurosci 27:4261-4272.

Roy NC, Bessaih T, Contreras D (2011) Comprehensive mapping of whisker-evoked responses reveals broad, sharply tuned thalamocortical input to layer 4 of barrel cortex. J Neurophysiol 105:2421-2437.

Sakata S, Harris KD (2009) Laminar structure of spontaneous and sensoryevoked population activity in auditory cortex. Neuron 64:404-418.

Sanchez-Vives MV, McCormick DA (2000) Cellular and network mechanisms of rhythmic recurrent activity in neocortex. Nat Neurosci 3:1027-1034.

Schubert D, Staiger JF, Cho N, Kötter R, Zilles K, Luhmann HJ (2001) Layer-specific intracolumnar and transcolumnar functional connectivity of layer V pyramidal cells in rat barrel cortex. J Neurosci 21:3580-3592.

Schubert D, Kötter R, Luhmann HJ, Staiger JF (2006) Morphology, electrophysiology and functional input connectivity of pyramidal neurons characterizes a genuine layer Va in the primary somatosensory cortex. Cereb Cortex 16:223-236.

Schubert D, Kötter R, Staiger JF (2007) Mapping functional connectivity in barrel-related columns reveals layer- and cell type-specific microcircuits. Brain Struct Funct 212:107-119.

Shepherd GM, Svoboda K (2005) Laminar and columnar organization of ascending excitatory projections to layer $2 / 3$ pyramidal neurons in rat barrel cortex. J Neurosci 25:5670-5679.

Shepherd GM, Stepanyants A, Bureau I, Chklovskii D, Svoboda K (2005) Geometric and functional organization of cortical circuits. Nat Neurosci 8:782-790.

Shu Y, Hasenstaub A, McCormick DA (2003) Turning on and off recurrent balanced cortical activity. Nature 423:288-293.

Silva LR, Amitai Y, Connors BW (1991) Intrinsic oscillations of neocortex generated by layer 5 pyramidal neurons. Science 251:432-435.

Staiger JF, Kötter R, Zilles K, Luhmann HJ (1999) Connectivity in the somatosensory cortex of the adolescent rat: an in vitro biocytin study. Anat Embryol (Berl) 199:357-365.

Steriade M, Contreras D (1995) Relations between cortical and thalamic cellular events during transition from sleep patterns to paroxysmal activity. J Neurosci 15:623-642.

Steriade M, Nuñez A, Amzica F (1993a) A novel slow ( $<1 \mathrm{~Hz}$ ) oscillation of neocortical neurons in vivo: depolarizing and hyperpolarizing components. J Neurosci 13:3252-3265.

Steriade M, Nuñez A, Amzica F (1993b) Intracellular analysis of relations between the slow $(<1 \mathrm{~Hz})$ neocortical oscillation and other sleep rhythms of the electroencephalogram. J Neurosci 13:3266-3283.

Steriade M, Contreras D, Curró Dossi R, Nuñez A (1993c) The slow $(<1$ $\mathrm{Hz}$ ) oscillation in reticular thalamic and thalamocortical neurons: scenario of sleep rhythm generation in interacting thalamic and neocortical networks. J Neurosci 13:3284-3299.

Steriade M, Amzica F, Neckelmann D, Timofeev I (1998) Spike-wave complexes and fast components of cortically generated seizures. II. Extra- and intracellular patterns. J Neurophysiol 80:1456-1479.

Telfeian AE, Connors BW (1998) Layer-specific pathways for the horizontal 
propagation of epileptiform discharges in neocortex. Epilepsia 39:700-708.

Theyel BB, Llano DA, Sherman SM (2010) The corticothalamocortical circuit drives higher-order cortex in the mouse. Nat Neurosci 13:84-88.

Thomson AM, Bannister AP (2003) Interlaminar connections in the neocortex. Cereb Cortex 13:5-14.

Thomson AM, Deuchars J (1997) Synaptic interactions in neocortical local circuits: dual intracellular recordings in vitro. Cereb Cortex 7:510-522.

Thomson AM, West DC, Wang Y, Bannister AP (2002) Synaptic connections and small circuits involving excitatory and inhibitory neurons in layers $2-5$ of adult rat and cat neocortex: triple intracellular recordings and biocytin labelling in vitro. Cereb Cortex 12:936-953.

Weiler N, Wood L, Yu J, Solla SA, Shepherd GM (2008) Top-down laminar organization of the excitatory network in motor cortex. Nat Neurosci 11:360-366.
Wilent WB, Contreras D (2004) Synaptic responses to whisker deflections in rat barrel cortex as a function of cortical layer and stimulus intensity. J Neurosci 24:3985-3998.

Wimmer VC, Bruno RM, de Kock CP, Kuner T, Sakmann B (2010) Dimensions of a projection column and architecture of VPM and POm axons in rat vibrissal cortex. Cereb Cortex 20:2265-2276.

Wong-Riley M (1979) Changes in the visual system of monocularly sutured or enucleated cats demonstrable with cytochrome oxidase histochemistry. Brain Res 171:11-28.

Yoshimura Y, Dantzker JL, Callaway EM (2005) Excitatory cortical neurons form fine-scale functional networks. Nature 433:868-873.

Yuste R, Tank DW, Kleinfeld D (1997) Functional study of the rat cortical microcircuitry with voltage-sensitive dye imaging of neocortical slices. Cereb Cortex 7:546-558. 